\title{
A new fitting function for GRB MeV spectra based on the internal shock synchrotron model
}

\author{
M. Yassine ${ }^{1,2,3}$, F. Piron ${ }^{1}$, F. Daigne ${ }^{4}$, R. Mochkovitch ${ }^{4}$, F. Longo $^{2,3}$, N. Omodei ${ }^{5}$, and G. Vianello ${ }^{5}$ \\ ${ }^{1}$ Laboratoire Univers et Particules de Montpellier, Université de Montpellier, CNRS/IN2P3, Montpellier, France \\ e-mail: piron@in2p3.fr \\ 2 Istituto Nazionale di Fisica Nucleare, Sezione di Trieste, 34127 Trieste, Italy \\ e-mail: francesco.longo@ts.infn.it \\ 3 Dipartimento di Fisica, Università di Trieste, 34127 Trieste, Italy \\ e-mail: mychbib@gmail.com \\ ${ }^{4}$ UPMC-CNRS, UMR7095, Institut d'Astrophysique de Paris, 75014 Paris, France \\ 5 W. W. Hansen Experimental Physics Laboratory, Kavli Institute for Particle Astrophysics and Cosmology, Department of Physics \\ and SLAC National Accelerator Laboratory, Stanford University, Stanford, CA 94305, USA
}

Received 5 November 2019 / Accepted 6 April 2020

\section{ABSTRACT}

\begin{abstract}
Aims. The physical origin of the gamma-ray burst (GRB) prompt emission is still a subject of debate. Internal shock models have been widely explored, owing to their ability to explain most of the high-energy properties of this emission phase. While the Band function or other phenomenological functions are commonly used to fit GRB prompt emission spectra, we propose a new parametric function that is inspired by an internal shock physical model. We use this function as a proxy of the model to compare it easily to GRB observations.

Methods. We built a parametric function that represents the spectral form of the synthetic bursts provided by our internal shock synchrotron model (ISSM). We simulated the response of the Fermi instruments to the synthetic bursts and fit the obtained count spectra to validate the ISSM function. Then, we applied this function to a sample of 74 bright GRBs detected by the Fermi GBM, and we computed the width of their spectral energy distributions around their peak energy. For comparison, we also fit the phenomenological functions that are commonly used in the literature. Finally, we performed a time-resolved analysis of the broadband spectrum of GRB 090926A, which was jointly detected by the Fermi GBM and LAT. This spectrum has a complex shape and exhibits a power-law component with an exponential cutoff at high energy, which is compatible with inverse Compton emission attenuated by gamma-ray internal absorption.

Results. This work proposes a new parametric function for spectral fitting that is based on a physical model. The ISSM function reproduces $81 \%$ of the spectra in the GBM bright GRB sample, versus 59\% for the Band function, for the same number of parameters. It gives also relatively good fits to the GRB 090926A spectra. The width of the MeV spectral component that is obtained from the fits of the ISSM function is slightly larger than the width from the Band fits, but it is smaller when observed over a wider energy range. Moreover, all of the 74 analyzed spectra are found to be significantly wider than the synthetic synchrotron spectra. We discuss possible solutions to reconcile the observations with the internal shock synchrotron model, such as an improved modeling of the shock microphysics or more accurate spectral measurements at $\mathrm{MeV}$ energies.
\end{abstract}

Key words. gamma-ray burst: general - radiation mechanisms: non-thermal

\section{Introduction}

Gamma-ray bursts (GRBs) were discovered more than fifty years ago, and they are the most electro-magnetic events ever observed in the Universe. They are brief flashes of high-energy radiation emitted by an ultra-relativistic collimated outflow which is thought to originate from a stellar-mass black hole formed by the merging of binary systems (Nakar 2007; D'Avanzo 2015) or the explosions of massive stars (Woosley \& Bloom 2006; Stanek et al. 2003; Kawabata et al. 2003; Hjorth et al. 2003, 2005; Bloom et al. 2002; Gehrels et al. 2005; Abbott et al. 2017). Emission from GRBs is observed in two successive phases, a short phase of intense radiation followed by a long-lived afterglow phase. While both emissions are essentially non thermal, the prompt phase is notably characterized by the irregular shape and the fast variability of its temporal profile. Despite substantial efforts in modeling the GRB prompt emission, different scenarios such as internal shocks (Rees \& Meszaros 1994), dissipative photospheres (Beloborodov \& Mészáros 2017) or reconnection above the photosphere (Giannios 2008; McKinney \& Uzdensky 2012; Sironi et al. 2015; Beniamini \& Granot 2016) have been proposed to explain its physical origin. Internal shock models have been explored in detail (Kobayashi et al. 1997; Daigne \& Mochkovitch 1998; Bošnjak et al. 2009; Daigne et al. 2011; Bošnjak \& Daigne 2014) owing to their ability to produce emissions from the visible to the $\mathrm{GeV}$ domain and to account for GRB observed properties such as their spectral evolution and the extreme variability seen in their light curves. In this class of models, the GRB relativistic outflow converts a fraction of its kinetic energy into internal energy through internal shocks, which occur when the distribution of the Lorentz factors in the flow is highly nonuniform. Part of the energy that is dissipated in the shocks is transferred to a fraction of the electrons that emit nonthermal synchrotron and inverse Compton radiations.

Since the launch of the Fermi satellite in June 2008, the GRB high-energy emission has been studied with great sensitivity. 
The Large Area Telescope (LAT, $20 \mathrm{MeV}-300 \mathrm{GeV}$, Atwood et al. 2009) has detected more than 180 GRBs (Ajello et al. 2019) thanks to its wide field of view $(2.4 \mathrm{sr})$, its large effective area $\left(\sim 0.9 \mathrm{~m}^{2}\right.$ above $\left.\sim 1 \mathrm{GeV}\right)$ and to the improved event reconstruction (Pass 8 hereafter) that has been implemented in 2015 (Atwood et al. 2013). The Gamma-ray Burst Monitor (GBM) is the second instrument onboard Fermi and it consists of 12 sodium iodide ( $\mathrm{NaI}, 8 \mathrm{keV}-1 \mathrm{MeV})$ and 2 bismuth germanate (BGO, $250 \mathrm{keV}-40 \mathrm{MeV}$ ) detectors placed around the Fermi spacecraft. The GBM monitors continuously a large portion of the sky $(9.5 \mathrm{sr})$, and it has detected more than 2600 GRBs so far (Narayana Bhat et al. 2016). Together, the GBM and the LAT cover more than seven decades in energy, hence they are the most suitable instruments currently in operations to study the broadband high-energy emission of GRBs.

The keV-MeV spectral component of GRBs, which is often attributed to synchrotron emission, is commonly fit by the phenomenological Band function (Band et al. 1993). Despite its ability to describe many of the GRB nonthermal spectra, this function has little physical grounds and is not suitable for a fair fraction of spectra (see, e.g., Gruber et al. 2014). The interpretation of the GRB spectral fit results faces another problem that was pointed out twenty years ago by Preece et al. (1998; see also Crider et al. 1997; Ghisellini et al. 2000; Burgess et al. 2015). In their analysis of CGRO/BATSE bursts, these authors came to the conclusion that most of the fit spectral slopes are too hard to be compatible with the expectations from the synchrotron theory at low energy, an issue that is now refered to as the "synchrotron line-of-death problem" (Ghisellini et al. 2000; Axelsson \& Borgonovo 2015; Burgess et al. 2015).

More recently, Yu et al. (2015) and Axelsson \& Borgonovo (2015) used the spectral-sharpness to show that the spectrum that is expected from an electron synchrotron model is wider than the Band spectra of most GRBs detected by the GBM, calling for a new physical interpretation of the keV-MeV spectral component. However, it should be noted that the theoretical spectrum considered in Yu et al. (2015) was essentially derived from a pure Maxwellian electron distribution, which does not account for the dynamical evolution of the electron and photon distributions in the GRB jet. In addition, the authors did not attempt to fit this theoretical model to the data, which might introduce instrumental biases in the comparison with the Band fit results. Direct fits of the synchrotron emission model to GRB prompt spectra have been performed by Zhang et al. (2016) and Burgess (2019), who showed that the line-of-death and spectralsharpness issues are likely artefacts due to the use of the Band function (see also Ronchi et al. 2020). Fitting the spectra with simpler versions of the synchrotron emission model or with empirical functions featuring a low-energy spectral break, especially on a broad energy range extending down to the X-ray and/or optical domain, appears able to reconcile the observations with the synchrotron theory as well, showing the expected transition from fast to slow cooling (Oganesyan et al. 2017, 2018, 2019; Ravasio et al. 2018, 2019).

In the same spirit, this work compares the predictions of an actual internal shock synchrotron model to the observations, using the version of the model described in Daigne \& Mochkovitch (1998), Bošnjak et al. (2009), Daigne et al. (2011), Bošnjak \& Daigne (2014). We simulated synthetic bursts provided by this model using the GBM and LAT detector responses. The characteristics of the synthetic bursts and our simulation procedure are described in Sect. 2. In Sect. 3 we present the functions used to fit the burst spectra, including a new fitting function (called ISSM hereafter) that is directly built from the synthetic spectra in the keV-MeV energy range. The spectral analysis of the synthetic bursts and the computation of their spectral width are reported in Sect. 4.

In Sect. 5, we apply the same set of fitting functions to a sample of 74 GBM bright GRBs. The data selection and the technique of identification of the best fit spectral model are presented, as well as a focus on the spectral parameters and sharpness obtained for the Band and ISSM functions. In Sect. 6 we revisit the spectral analysis of GRB 090926A using the new ISSM function. This burst was bright in the GBM and LAT instruments, and it exhibits fast variability above $100 \mathrm{MeV}$ during the $\mathrm{keV}-\mathrm{MeV}$ prompt emission. As reported in Yassine et al. (2017; Y17 hereafter), it constitutes an ideal case to test the internal shock model from $\mathrm{keV}$ to $\mathrm{GeV}$ energies. Finally, we discuss our results in Sect. 7 and give our conclusions in Sect. 8.

\section{Simulation of the synthetic bursts}

\subsection{The internal shock model}

The version of the internal shock model that we used is able to reproduce most of the GRB properties, in particular the variability timescales and the shape of the GRB light curves (Daigne \& Mochkovitch 1998). In this model, the GRB outflow consists of a set of solid layers which move at different Lorentz factors, whose collisions mimic the propagation of an internal shock wave along the GRB jet. Each GRB is characterized by its redshift, duration and kinetic energy, and by a Lorentz factor profile. The model also assumes that some fraction $\epsilon_{B}$ of the energy dissipated in the shocks is transferred to the magnetic field, and that a fair fraction $\epsilon_{\mathrm{e}}$ is injected into a small part $\zeta$ of accelerated electrons. The energy distribution of the accelerated electrons is a power law with a slope $-p$, which is set to a value ranging from -2.9 to -2.3 . This adopted interval for the index of the electron distribution corresponds to a typical high-energy spectral index $\beta=-(p / 2+1)$ between 2.15 and 2.45 which is consistent with observations. In addition to the GRB outflow dynamics, the model accounts for the main radiative processes at high energy. The numerical code that simulates the shock dynamics was coupled to a radiative code, which follows the evolution of the electron and photon distributions in order to produce realistic light curves and spectra from $\mathrm{keV}$ to $\mathrm{GeV}$ energies in the observer frame (Bošnjak et al. 2009). The radiative processes include the synchrotron emission from the accelerated electrons and the inverse Compton (IC) scatterings in the Thomson and Klein-Nishina regimes. Synchrotron self-absorption at low energy and photon-photon annihilation at high energy are also accounted for.

\subsection{Characteristics of the bursts}

The synthetic burst that we considered corresponds to the case B of Bošnjak \& Daigne (2014; BD14 hereafter) owing to its typical kinetic energy, $E_{\mathrm{k}}=10^{54} \mathrm{erg}$, and to its brightness in the LAT energy range. The burst is long, with a duration of $15 \mathrm{~s}$, and it is bright during the first $6 \mathrm{~s}$ only. The microphysical parameters describing the electron distribution are $\epsilon_{\mathrm{e}}=1 / 3$, $p=2.7$, and a varying fraction $\zeta$ of accelerated particles. The low magnetic energy density $\left(\epsilon_{B}=10^{-3}\right)$ enhances the IC component and makes this burst an interesting candidate for a LAT detection. The burst has an isotropic equivalent energy $E_{\gamma, \text { iso }}=$ $1.35 \times 10^{52} \mathrm{erg}$, with $1.26 \times 10^{52} \mathrm{erg}$ in the synchrotron component and $0.09 \times 10^{52} \mathrm{erg}$ in the IC component. The low and 

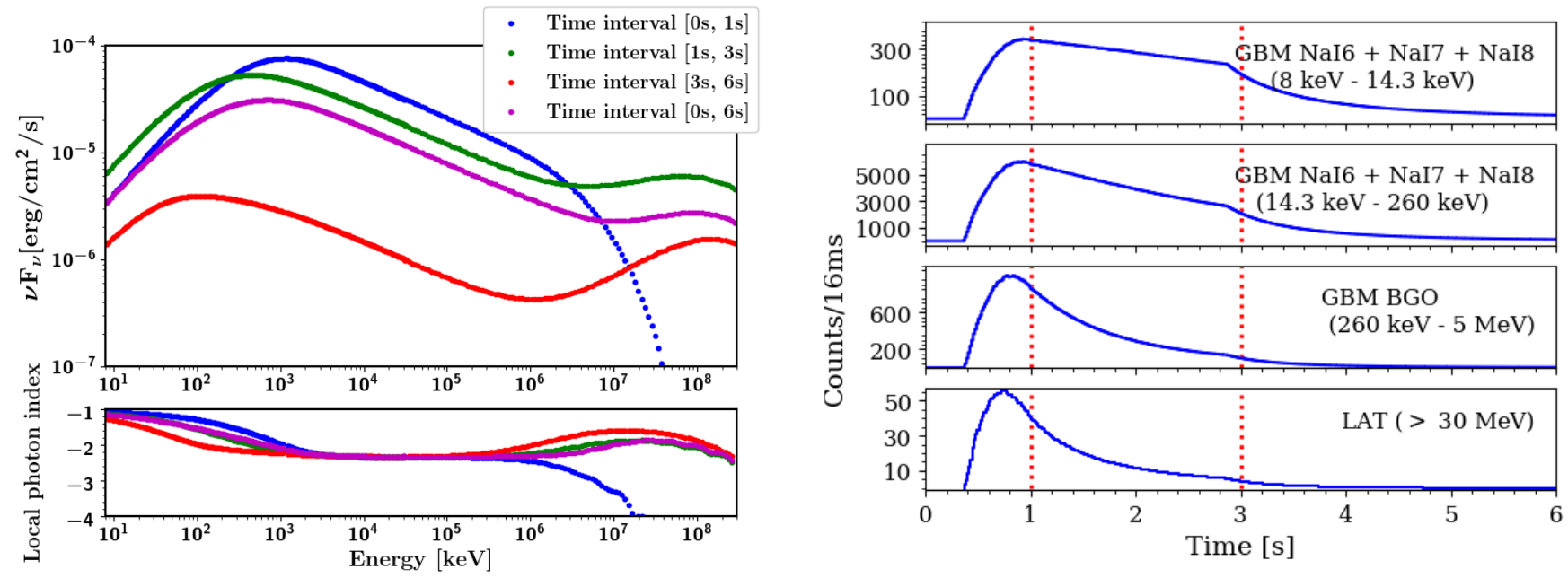

Fig. 1. Left: spectral energy distributions of the synthetic burst GRB_B010 and local photon index in the four time intervals [0 s, $1 \mathrm{~s}]$, [1 s, $3 \mathrm{~s}]$, [3 s, $6 \mathrm{~s}]$ and [0 s, $6 \mathrm{~s}]$. Right: multi-detector light curves of GRB_B010: summed counts in two energy ranges of GBM/NaI detectors (first two panels), in the GBM/BGO energy range (third panel) and using the largest LAT energy range ( $>30 \mathrm{MeV})$ (bottom panel). The red dashed lines show the boundaries of the three time intervals $[0 \mathrm{~s}, 1 \mathrm{~s}],[1 \mathrm{~s}, 3 \mathrm{~s}]$ and $[3 \mathrm{~s}, 6 \mathrm{~s}]$.

high-energy indices of the synchrotron spectrum are $\sim-1.1$ and $\sim-2.4$, respectively.

We placed the synthetic burst at a low redshift $z=0.07$ as an easy way to increase the observed flux and to produce a large number of simulated counts in the Fermi instruments. As we explain further below, this allowed us to characterize with high accuracy and unambiguously the properties of the burst emission folded with the instrument responses. As a result, the fluence of the synthetic burst is $5.4 \times 10^{-4} \mathrm{erg} \mathrm{cm}^{-2}$ between $10 \mathrm{keV}$ and $1 \mathrm{MeV}$ during the first $6 \mathrm{~s}$. This would be a very rare event among the GRBs that have been jointly detected by the GBM and the LAT, whose fluence varies from $5 \times 10^{-8} \mathrm{erg} \mathrm{cm}^{-2}$ to $\sim 3 \times 10^{-4} \mathrm{erg} \mathrm{cm}^{-2}$ in the same energy range (Ackermann et al. 2013). In order to consider more realistic situations, two other synthetic bursts were created by dividing the simulated emission flux by 10 and 100 .

In the following, the three synthetic bursts are denoted by GRB_B001,GRB_B010, and GRB_B100 in order of decreasing flux. We split the light curve of each of these bursts in three time intervals, $[0 \mathrm{~s}, 1 \mathrm{~s}],[1 \mathrm{~s}, 3 \mathrm{~s}]$ and $[3 \mathrm{~s}, 6 \mathrm{~s}]$. The upper panel in the left part of Fig. 1 shows the corresponding spectral energy distributions (SEDs) of GRB_B010, in addition to the SED of this burst during the total time interval $[0 \mathrm{~s}, 6 \mathrm{~s}]$. The lower panel shows the evolution with energy of the local photon index $\Gamma(E)$, which we calculated numerically as the logarithmic derivative of the differential photon spectrum $F=\mathrm{d} N / \mathrm{d} E$ with respect to the logarithmic energy, $\Gamma(E)=\partial \ln (F) / \partial \ln (E)$.

\subsection{Simulation procedure}

We simulated the signal of the synthetic bursts as it would be observed by the GBM or the LAT by performing a convolution of the GRB differential photon spectra $\mathrm{d} N / \mathrm{d} E$ with the corresponding detector response matrix (DRM). The DRM is defined as the detector effective area $A_{\text {eff }}(E)$ multiplied by its energy redistribution function $D\left(E, E^{\prime}\right)$, where $E$ and $E^{\prime}$ stand for true and measured photon energy, respectively. The mean number of counts in the interval of measured energy $\left[E_{\min }^{\prime}, E_{\max }^{\prime}\right]$ is given by:

$N=T_{\mathrm{obs}} \int_{E_{\min }^{\prime}}^{E_{\max }^{\prime}} \mathrm{d} E^{\prime} \int_{0}^{+\infty} \frac{\mathrm{d} N}{\mathrm{~d} E}(E) A_{\text {eff }}(E) D\left(E, E^{\prime}\right) \mathrm{d} E$ where $T_{\text {obs }}$ is the time exposure. For this computation, we used the DRMs of the four GBM detectors (NaI6, 7, 8 and BGO1) that have seen GRB 090926A and the DRM of the LAT produced by the gtrspgen ${ }^{1}$ tool available at the Fermi Science Support Cen$t^{2}{ }^{2}$. The simulation of the synthetic bursts was performed with the XSPEC software ${ }^{3}$ (version 12.8.2), which generates Poisson counts of detected photons. For simplicity, we did not add any background to the burst signal since it has a negligible effect owing to the large fluence of the simulated bursts. The multidetector light curve of the synthetic burst GRB_B010 is shown in the right part of Fig. 1.

\section{Spectral models}

The GRB spectra that we analyzed were fitted with several phenomenological functions that are commonly found in the literature, and with a new parametric function that is built from the synthetic spectra. All of the functions presented below are normalized by an amplitude parameter $A$, in units of $\mathrm{cm}^{-2} \mathrm{~s}^{-1} \mathrm{keV}^{-1}$.

\subsection{Phenomenological models}

\subsubsection{Band function}

The Band function (Band et al. 1993) is often used to fit the $\mathrm{keV}-\mathrm{MeV}$ spectrum of GRBs. It is composed of two smoothly connected power laws with four parameters $A_{\mathrm{Band}}, \alpha, \beta$ and $E_{\mathrm{p}}$, and it is defined as:

$$
\frac{\mathrm{d} N_{\text {Band }}}{\mathrm{d} E}(E)=A_{\text {Band }} \begin{cases}\left(\frac{E}{100 \mathrm{keV}}\right)^{\alpha} \exp \left[-\frac{E(2+\alpha)}{E_{\mathrm{p}}}\right], & E \leq E_{\mathrm{b}}=E_{\mathrm{p}} \frac{\alpha-\beta}{2+\alpha} \\ \left(\frac{E}{100 \mathrm{keV}}\right)^{\beta}\left[\frac{E_{\mathrm{p}}}{100 \mathrm{keV}} \frac{\alpha-\beta}{2+\alpha}\right]^{\alpha-\beta} & \\ \times \exp [\beta-\alpha], & E>E_{\mathrm{b}}=E_{\mathrm{p}} \frac{\alpha-\beta}{2+\alpha} .\end{cases}
$$

\footnotetext{
1 https://fermi.gsfc.nasa.gov/ssc/data/analysis/ scitools/help/gtrspgen.txt

2 https://fermi.gsfc.nasa.gov/ssc/data/analysis/

scitools/overview.html

3 https://heasarc.gsfc.nasa.gov/xanadu/xspec/
} 
The local photon index of this function reads:

$\Gamma_{\text {Band }}(E)= \begin{cases}\alpha-\frac{(2+\alpha)}{E_{\mathrm{p}}} E, & E \leq E_{\mathrm{b}} \\ \beta, & E>E_{\mathrm{b}}\end{cases}$

\subsubsection{Logarithmic parabola and variants}

The log-parabola function (LP hereafter) has three free parameters, i.e. one less than the Band function. Massaro et al. (2010) suggested using it to fit GRB spectra and it is expressed as:

$\frac{\mathrm{d} N_{\mathrm{LP}}}{\mathrm{d} E}(E)=A_{\mathrm{LP}}\left(\frac{E}{E_{0}}\right)^{-\gamma-\beta \log \left(E / E_{0}\right)}$

where $E_{0}$ is a fixed reference energy. The local photon index is a function of the spectral parameters $\gamma$ and $\beta$ :

$\Gamma_{\mathrm{LP}}(E)=-\gamma-2 \beta \log \left(\frac{E}{E_{0}}\right)$

and the LP peak energy is $E_{\mathrm{p}}=E_{0} \times 10^{\frac{2-\gamma}{2 \beta}}$. The LP function is characterized by its continuous curvature, unlike the Band function. Its symmetric shape implies that the spectral parameter reconstruction is driven by the low-energy data, where most of the photon statistics is recorded. In order to gain some latitude at high energies, we modified the function to freeze the local photon index above a break energy $E_{\mathrm{b}}$. As a result, the modified logarithmic parabola, denoted by LP1 hereafter, has four free parameters:

$\frac{\mathrm{d} N_{\mathrm{LP} 1}}{\mathrm{~d} E}(E)=A_{\mathrm{LP} 1} \begin{cases} & \left(\frac{E}{E_{0}}\right)^{-\gamma-\beta \log \left(E / E_{0}\right)}, \\ \left(\frac{E_{\mathrm{b}}}{E_{0}}\right)^{-\gamma-\beta \log \left(E_{\mathrm{b}} / E_{0}\right)} & \\ \times\left(\frac{E}{E_{\mathrm{b}}}\right)^{-\gamma-2 \beta \log \left(E_{\mathrm{b}} / E_{0}\right)}, & \end{cases}$

We introduced a similar modification at low energies, which relaxes the dependency of the spectral fit around the peak energy on the low-energy data. The corresponding modified logarithmic parabola, denoted by LP2 hereafter, has five free parameters:

$$
\frac{\mathrm{d} N_{\mathrm{LP} 2}}{\mathrm{~d} E}(E)=A_{\mathrm{LP} 2}\left\{\begin{array}{lr}
\left(\frac{E_{\mathrm{b}}^{\prime}}{E_{0}}\right)^{-\gamma-\beta \log \left(E_{\mathrm{b}}^{\prime} / E_{0}\right)} \times\left(\frac{E}{E_{\mathrm{b}}^{\prime}}\right)^{-\gamma-2 \beta \log \left(E_{\mathrm{b}}^{\prime} / E_{0}\right)}, \quad E \leq E_{\mathrm{b}}^{\prime} \\
\left(\frac{E}{E_{0}}\right)^{-\gamma-\beta \log \left(E / E_{0}\right)}, \quad E_{\mathrm{b}}^{\prime} \leq E \leq E_{\mathrm{b}} \\
\left(\frac{E_{\mathrm{b}}}{E_{0}}\right)^{-\gamma-\beta \log \left(E_{\mathrm{b}} / E_{0}\right)} \times\left(\frac{E}{E_{\mathrm{b}}}\right)^{-\gamma-2 \beta \log \left(E_{\mathrm{b}} / E_{0}\right)}, \quad E>E_{\mathrm{b}}
\end{array}\right.
$$

\subsection{3. (Broken) power law with exponential cutoff}

For the spectral analysis of GRB 090926A presented in Sect. 6, which extends to the LAT energy range, we adopted either a power law with exponential cutoff (CUTPL) or a broken power law with exponential cutoff (CUTBPL). The CUTPL function is expressed as:

$$
\frac{\mathrm{d} N_{\text {CUTPL }}}{\mathrm{d} E}(E)=A_{\text {CUTPL }}\left(\frac{E}{E_{0}}\right)^{\lambda} \exp \left(-\frac{E}{E_{\mathrm{f}}}\right)
$$

which has three free parameters $A_{\text {CUTPL }}, \lambda$ and the folding energy $E_{\mathrm{f}}$ of the exponential cutoff, and a fixed reference energy $E_{0}$. The CUTBPL function is expressed as:

$$
\frac{\mathrm{d} N_{\text {CUTBPL }}}{\mathrm{d} E}(E)=A_{\text {CUTBPL }} \begin{cases}\left(\frac{E}{E_{0}}\right)^{\gamma_{0}} \exp \left(-\frac{E}{E_{\mathrm{f}}}\right), & E \leq E_{\mathrm{b}} \\ \left(\frac{E_{\mathrm{b}}}{E_{0}}\right)^{\gamma_{0}}\left(\frac{E}{E_{\mathrm{b}}}\right)^{\gamma} \exp \left(-\frac{E}{E_{\mathrm{f}}}\right), & E>E_{\mathrm{b}}\end{cases}
$$

where $E_{\mathrm{b}}$ is the break energy, $\gamma_{0}$ and $\gamma$ are the photon index below and above $E_{\mathrm{b}}$, respectively. As explained in Y17, the break energy and the photon spectral index below the break were fixed to $E_{\mathrm{b}}=200 \mathrm{keV}$ and $\gamma_{0}=+4$ in order to cancel the contribution of the power-law component at low energies, as for instance expected from an inverse Compton spectral component that would extend the synchrotron spectrum at high energies only. As a result, the CUTBPL function has the same number of free parameters as the CUTPL function.

\subsection{The ISSM spectral model}

In order to build a function that is representative of the synchrotron spectral component of the synthetic bursts, we fitted their local photon index as a function of energy with the following parameterization:

$\Gamma(E)=\frac{\partial \ln (F)}{\partial \ln (E)}=-a+\frac{b}{E+c}$

where $a, b, c$ are free parameters. This parameterization adequately fits the local photon index of GRB_B001 in the four time intervals as shown in the left panel of Fig. 2. The right panel of this figure shows that it is also suitable for different configurations of the model presented in BD14. Note that the synthetic bursts using various assumptions for the microphysics in the emission region do not have the same low-energy photon index: $\sim 1.5$ for case $\mathrm{A}$ as expected for the standard fast cooling synchrotron spectrum, and -1.1 to -0.75 for case $\mathrm{B}$. When integrating Eq. (10), one gets:

$F(E)=F\left(E_{\mathrm{r}}\right) \exp \left[-a \ln \left(\frac{E}{E_{\mathrm{r}}}\right)\right] \exp \left[\frac{b}{c} \ln \left(\frac{E\left(E_{\mathrm{r}}+c\right)}{E_{\mathrm{r}}(E+c)}\right)\right]$

where the reference energy $E_{\mathrm{r}}$ is related to the constant of integration. From this parameterization, the asymptotic spectral indices towards low and high energies can be easily obtained as $\alpha=\frac{b}{c}-a$ and $\beta=-a$, respectively. Finally, defining the SED peak energy $E_{\mathrm{p}}$ as the solution of $\Gamma(E)=-2$ :

$E_{\mathrm{p}}=-c\left(\frac{2+\alpha}{2+\beta}\right)$,

one can rewrite Eq. (11) to obtain a new expression, denoted by ISSM hereafter:

$\frac{\mathrm{d} N_{\text {ISSM }}}{\mathrm{d} E}(E)=\frac{A_{\text {ISSM }}}{\left[1-\frac{E_{\mathrm{p}}}{E_{\mathrm{r}}}\left(\frac{2+\beta}{2+\alpha}\right)\right]^{\beta-\alpha}} \times\left(\frac{E}{E_{\mathrm{r}}}\right)^{\alpha}\left[\frac{E}{E_{\mathrm{r}}}-\frac{E_{\mathrm{p}}}{E_{\mathrm{r}}}\left(\frac{2+\beta}{2+\alpha}\right)\right]^{\beta-\alpha}$

which has four parameters $A_{\mathrm{ISSM}}, \alpha, \beta, E_{\mathrm{p}}$. It is important to note that $E_{\mathrm{r}}$ is a fixed reference energy which is chosen as the energy at which the flux normalization is defined:

$\frac{\mathrm{d} N_{\text {ISSM }}}{\mathrm{d} E}\left(E_{\mathrm{r}}\right)=A_{\text {ISSM }}$.

In other words, different choices of $E_{\mathrm{r}}$ only affect the flux normalization parameter $A_{\text {ISSM }}$ and not the shape of the ISSM function. The local photon index is given by:

$\Gamma_{\text {ISSM }}(E)=\alpha+(\beta-\alpha) \frac{E}{E-E_{\mathrm{p}}\left(\frac{2+\beta}{2+\alpha}\right)}$.

The four parameters of the ISSM (flux normalization, SED peak energy and asymptotic slopes) resemble those of the Band function. The local photon index $\Gamma_{\text {ISSM }}(E)$ decreases continuously 

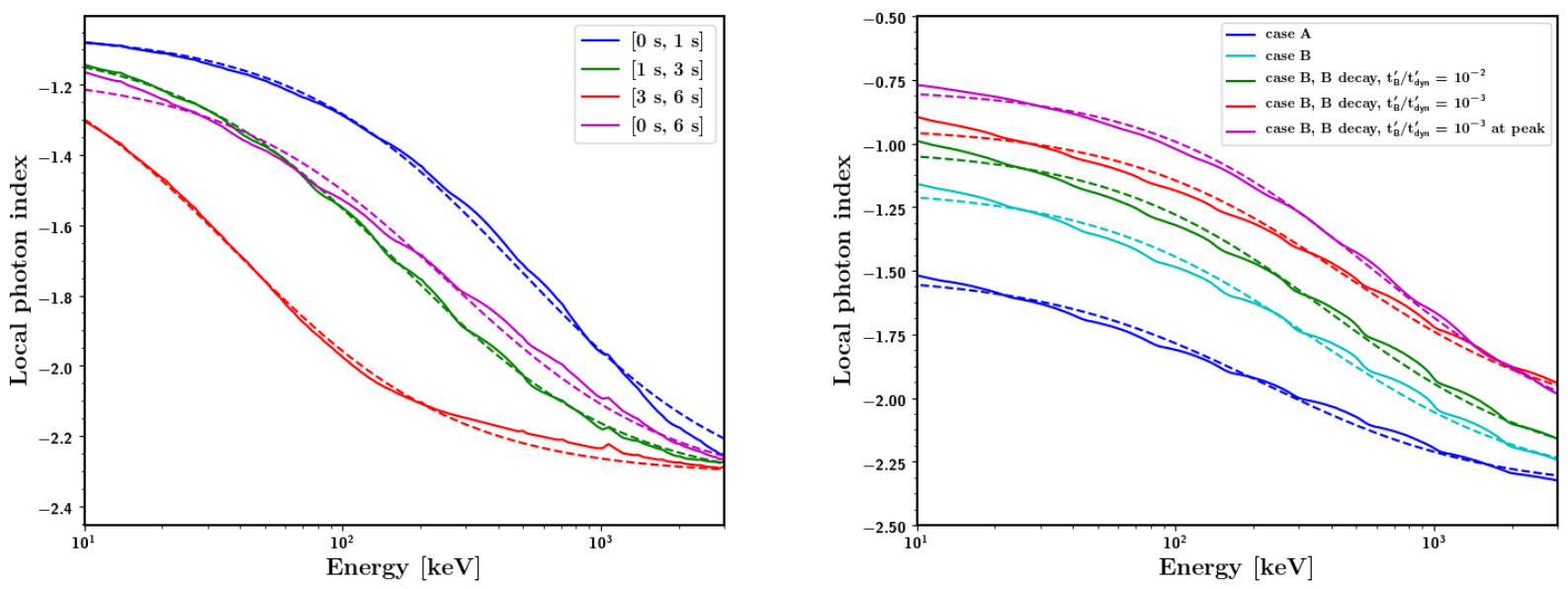

Fig. 2. Left: least-square fit (dashed lines) of Eq. (10) to the local photon index (thick lines) in the keV-MeV range for the four time intervals of the synthetic burst GRB_B001. Right: least-square fit (dashed line) to the local photon index (solid line) in the keV-MeV range for several synthetic bursts: time integrated spectrum of two reference cases presented in BD14, case A (blue) and B (cyan) with a varying fraction of accelerated electrons and $p=2.7$. Case A corresponds to the most standard fast cooling synchrotron spectrum, and case B to a modified synchrotron spectrum affected by inverse Compton scaterring in the Klein-Nishina regime. In addition, preliminary calculation of the same case B taking into account a magnetic field decay in the emission region are also presented (taken from Daigne $\&$ Bošnjak, in prep.) with a time scale of the decay $t_{B}^{\prime} / t_{\mathrm{dyn}}^{\prime}=10^{-2}$ (green) or $10^{-3}$ (red), where $t_{\mathrm{dyn}}^{\prime}$ is the dynamical timescale. Finally, the same case is also shown for a time interval of $0.25 \mathrm{~s}$ around the peak of the light curve (magenta).

with energy and the ISSM function is continuously curved unlike the Band function, and unlike simplified versions of the synchrotron model based on pure power-law energy distributions of the accelerated electrons. In the framework of our internal shock synchrotron model, the spectral curvature arises essentially from the superposition of instantaneous electron synchrotron spectra which vary significantly within the time intervals considered by the observer, owing to the dynamical evolution in the shock region. While we only tested the ISSM function on a simple, single-pulse burst, we are confident that it can also represent complex burst spectra resulting from various distributions of the Lorentz factor. Indeed, in most cases, complex bursts can be interpreted in terms of a succession of individual pulses so that time-dependent spectra of complex bursts can likely be fitted in the same way. Moreover, BD14 actually explored in detail how the observed emission of a single pulse depends on the various physical parameters of the internal shock model. Their study shows that the assumptions about the dynamics (Lorentz factor, kinetic energy flux, etc.) affect the pulse light curve but have little effect on the shape of the spectrum.

\section{Spectral analysis of the synthetic bursts}

We first focused our study of the three synthetic bursts in the GBM energy range ( $8 \mathrm{keV}$ to $40 \mathrm{MeV}$ ). The four phenomenological functions and the ISSM function were used to fit the spectra of the synthetic bursts in the four time intervals $[0 \mathrm{~s}, 1 \mathrm{~s}],[1 \mathrm{~s}$, $3 \mathrm{~s}],[3 \mathrm{~s}, 6 \mathrm{~s}]$ and $[0 \mathrm{~s}, 6 \mathrm{~s}]$ using the XSPEC software. The reference energy $E_{0}$ in Eqs. (4), (6) and (7) was fixed to $500 \mathrm{keV}$. For simplicity, the reference energy in Eq. (13), which relates to the flux normalization, was fixed to the true peak energy of the synthetic spectra: $E_{\mathrm{r}}=1150,478,114$ and $745 \mathrm{keV}$ for the time intervals [0s, $1 \mathrm{~s}],[1 \mathrm{~s}, 3 \mathrm{~s}],[3 \mathrm{~s}, 6 \mathrm{~s}]$ and [0 s, $6 \mathrm{~s}]$, respectively. To compare the quality of the fits between the different functions, we defined the following quality factor $Q$ that mimics a reduced $\chi^{2}$ :

$Q=\frac{1}{n-n_{\mathrm{par}}} \sum_{i=1}^{n}\left(\frac{\Gamma\left(E_{\mathrm{i}}\right)-s\left(E_{\mathrm{i}}\right)}{\sigma_{\mathrm{i}}}\right)^{2}$ where $\Gamma\left(E_{\mathrm{i}}\right)$ is the local photon index of the fit function and $s\left(E_{\mathrm{i}}\right)$ is the true index of the synthetic spectrum at energy $E_{\mathrm{i}}$. The error $\sigma_{\mathrm{i}}$ on $\Gamma\left(E_{\mathrm{i}}\right)$ is obtained by propagating the errors of the $n_{\mathrm{par}}$ fitted function parameters.

The spectral analyses were performed using the Castor fit statistic $^{4}\left(C_{\text {stat }}\right)$ for Poisson distributed total counts of the burst. The $C_{\text {stat }}$ values obtained from the fits of the three synthetic burst spectra are reported in Table 1. The ISSM function has the lowest $C_{\text {stat }}$ value in most of the time intervals especially for the synthetic burst with the highest flux value, for instance, GRB_B001. For GRB_B100, all functions yield similar $C_{\text {stat }}$ values, meaning that the fits are of similar quality as a result of the low photon statistics for this faint burst. Figure 3 shows the SEDs and local photon index of the GRB_B010 burst in the time interval [1 s, $3 \mathrm{~s}$, as obtained from the fits with the five spectral functions. As can be seen from this figure, both the SED and the local photon index are not reproduced by the Band function fit, in particular around and above the peak energy. The fit quality of the LP function is even worse due to the linear dependency of its local photon index with energy, which is not adequate at low and high energies. The LP1 and LP2 functions provide better fits and their parameters are not constrained for the three bursts in all the time intervals. Finally, Fig. 3 shows that the ISSM function has the lowest $Q$ value among all fit functions, which is expected from this model that was built directly from the synthetic spectra.

By nature, the ISSM function reproduces the keV-MeV spectra of the synthetic bursts simulated with the internal shock synchrotron model. It has the same number of free parameters as the Band function, which is commonly used to fit the prompt highenergy spectrum of GRBs. Therefore, before applying these functions to real GRB observations (see Sect. 5), it is worth comparing their shapes in detail. Tables A.1-A.3 show the parameters of the Band and ISSM fits to GRB_B001, GRB_B010, and GRB_B100, respectively. The asymptotic low-energy index $\alpha$ of the ISSM function is found to be larger than that of the Band function, while the high-energy index $\beta$ is smaller.

\footnotetext{
4 See https://heasarc.gsfc.nasa.gov/docs/xanadu/xspec/
} xspec11/manual/node57.html 
Table 1. $C_{\text {stat }}$ values of the spectral fits of the three synthetic bursts, performed with the five functions: Band, LP, LP1, LP2 and ISSM.

\begin{tabular}{llccccc}
\hline \hline \multirow{2}{*}{ Synthetic GRB } & \multirow{2}{*}{ Model } & d.o.f. & \multicolumn{4}{c}{$C_{\text {stat }}$ for time intervals: } \\
\cline { 4 - 7 } & & & {$[0-1] \mathrm{s}$} & {$[1-3] \mathrm{s}$} & {$[3-6] \mathrm{s}$} & {$[0-6] \mathrm{s}$} \\
\hline & Band & 473 & 603 & 906 & 486 & 1403 \\
& LP & 474 & 768 & 677 & 615 & 631 \\
GRB_B001 & LP1 & 473 & 768 & 569 & 615 & 589 \\
& LP2 & 472 & 526 & 540 & 558 & 570 \\
& ISSM & 473 & 486 & 498 & 452 & 638 \\
\hline & Band & 473 & 458 & 539 & 447 & 578 \\
& LP & 474 & 470 & 559 & 455 & 469 \\
GRB_B010 & LP1 & 473 & 470 & 534 & 455 & 469 \\
& LP2 & 472 & 439 & 525 & 455 & 466 \\
& ISSM & 473 & 441 & 523 & 443 & 484 \\
\hline & Band & 473 & 465 & 446 & 359 & 461 \\
& LP & 474 & 464 & 445 & 364 & 447 \\
GRB_B100 & LP1 & 473 & 464 & 445 & 364 & 447 \\
& LP2 & 472 & 462 & 442 & 376 & 447 \\
& ISSM & 473 & 463 & 444 & 360 & 449 \\
\hline
\end{tabular}
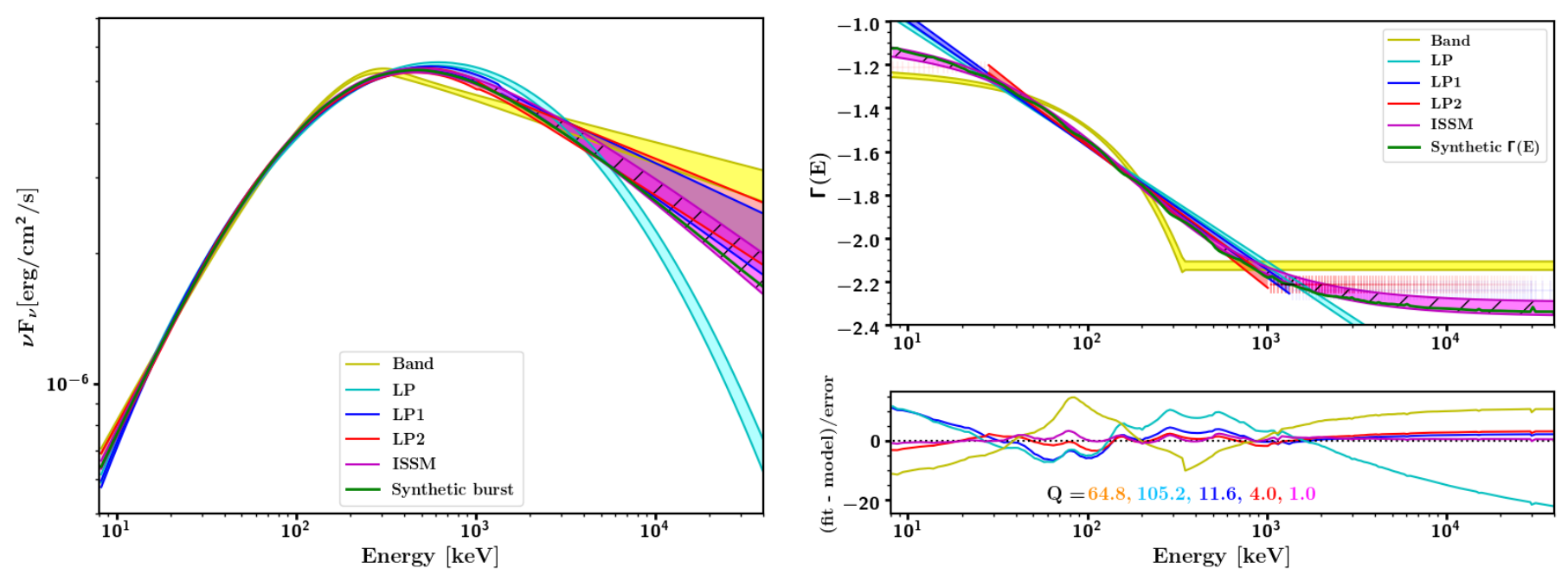

Fig. 3. Left: SEDs of the GRB_B010 synthetic burst in the time interval $[1 \mathrm{~s}, 3 \mathrm{~s}]$, from fits with the five spectral functions. The fit with the ISSMfunction is presented by the hatched magenta line. Right: local photon index as a function of the photon energy. The fit quality factor $Q$ of the five functions is given in the bottom panel.

Interestingly, the peak energies of the synthetic bursts are estimated with much greater accuracy with the ISSM function than with the Bandfunction, which underestimates them by $\sim 36 \%$. Furthermore, we compared the spectral width of the two functions, following $\mathrm{Yu}$ et al. (2015) who proposed a method to calculate the SED sharpness around its peak energy. We did not consider the alternate measure of the spectral width proposed by Axelsson \& Borgonovo (2015), which is defined as $W=\log \left(E_{2} / E_{1}\right)$, where $E_{1}$ and $E_{2}$ are the energy bounds of the SED full width at half maximum. The spectral sharpness angle defined by $\mathrm{Yu}$ et al. (2015) is computed from the triangle defined by the vertices at $E_{\mathrm{p}} / 10, E_{\mathrm{p}}$, and $3 E_{\mathrm{p}}$. To compute this angle and its asymmetrical errors accurately, we performed Monte Carlo simulations using the fit parameters and their covariance matrix, assuming that their distribution is a multivariate Gaussian. This process was repeated 1000 times for each time interval and for each of the two bright synthetic bursts GRB_B001 and GRB_B010. The spectral sharpness angle was chosen as the maximum probability value (MPV) of the distribution obtained from the 1000 realizations. The errors on the angle were calculated from the $68 \%$ confidence intervals on each side of the MPV. The results of this analysis are reported in Table 2, which confirms that the ISSM function reproduces the spectral width of the synthetic bursts better than the Band function.

For the sake of completeness, we carried out broadband spectral analyses of the brightest synthetic burst (GRB_B001) in the two time intervals $[1 s-3 s]$ and $[3 s-6 s]$, where the inverse Compton spectral component is prominent. We used the CUTPL model to fit this high-energy spectral component and fixed the reference energy $E_{0}$ to $10 \mathrm{GeV}$ in Eq. (8). This value is close to the decorrelation energy and thus minimizes the correlation between the CUTPL parameters. Despite its brightness in the LAT energy range, the inverse Compton component of GRB_B001 peaks at $\sim 100 \mathrm{GeV}$, where few simulated events are recorded. We multiplied artificially the LAT effective detection area by 100 to get rid of these statistical limitations and to check whether the adopted model is able to capture all features in the internal shock model spectra. The fit results obtained with the ISSM + CUTPL 
M. Yassine et al.: A new fitting function for GRB MeV spectra based on the internal shock synchrotron model

Table 2. Spectral sharpness angle (in degrees) from the Band and ISSM fits to the synthetic bursts GRB_B001 and GRB_B010.

\begin{tabular}{llcccc}
\hline \hline \multirow{2}{*}{ Synthetic GRB } & \multirow{2}{*}{ Model } & \multicolumn{4}{c}{ Time interval } \\
\cline { 3 - 6 } & & {$[0-1] \mathrm{s}$} & {$[1-3] \mathrm{s}$} & {$[3-6] \mathrm{s}$} & {$[0-6] \mathrm{s}$} \\
\hline & Synthetic & 142.3 & 145.9 & 145.5 & 148.9 \\
& Band & $137.7_{-0.2}^{+0.4}$ & $140.0_{-0.1}^{+0.2}$ & $142.7_{-0.3}^{+0.6}$ & $142.0_{-0.1}^{+0.2}$ \\
GRB_B001 & ISSM & $143.5_{-0.5}^{+0.5}$ & $145.7_{-0.3}^{+0.3}$ & $145.8_{-1.0}^{+1.3}$ & $148.6_{-0.3}^{+0.4}$ \\
\hline & Synthetic & 142.3 & 145.9 & 145.5 & 148.9 \\
& Band & $139.1_{-1.2}^{+0.9}$ & $139.9_{-0.4}^{+0.7}$ & $145.1_{-1.4}^{+1.8}$ & $142.6_{-0.6}^{+0.4}$ \\
GRB_B010 & ISSM & $143.6_{-1.3}^{+1.8}$ & $146.5_{-1.2}^{+1.1}$ & $150.6_{-4.7}^{+4.7}$ & $148.9_{-0.8}^{+1.3}$ \\
\hline
\end{tabular}

Table 3. Results of the ISSM + CUTPL fits to the synthetic burst GRB_B001 during the time intervals [ $1 \mathrm{~s}-3 \mathrm{~s}$ ] and [3 s-6s].

\begin{tabular}{llcc}
\hline \hline \multirow{2}{*}{$\begin{array}{l}\text { Spectral } \\
\text { component }\end{array}$} & \multirow{2}{*}{ Fit results } & \multicolumn{2}{c}{ Time interval } \\
\cline { 3 - 4 } ISSM & & {$[1-3] \mathrm{s}$} & {$[3-6] \mathrm{s}$} \\
& $E_{\mathrm{p}}(\mathrm{keV})$ & $458 \pm 4$ & $119 \pm 3$ \\
& $\alpha$ & $-1.09 \pm 0.01$ & $-1.03 \pm 0.05$ \\
& $\beta$ & $-2.354 \pm 0.003$ & $-2.321 \pm 0.004$ \\
& $A_{\mathrm{MeV}}\left(\mathrm{keV}^{-1} \mathrm{~cm}^{-2} \mathrm{~s}^{-1}\right)$ & $0.145 \pm 0.001$ & $0.193 \pm 0.001$ \\
\hline CUTPL & $\lambda$ & $-1.51 \pm 0.06$ & $-1.28 \pm 0.07$ \\
& $E_{\mathrm{f}}(\mathrm{GeV})$ & $165 \pm 71$ & $172 \pm 91$ \\
& $A_{\mathrm{GeV}}\left(\mathrm{keV}^{-1} \mathrm{~cm}^{-2} \mathrm{~s}^{-1}\right)$ & $(167.4 \pm 11.5) \times 10^{-13}$ & $(39.0 \pm 3.5) \times 10^{-13}$ \\
\hline ISSM + CUTPL & $C_{\mathrm{stat} / \mathrm{d} .0 . f .}$ & $533 / 510$ & $502 / 510$ \\
\hline
\end{tabular}
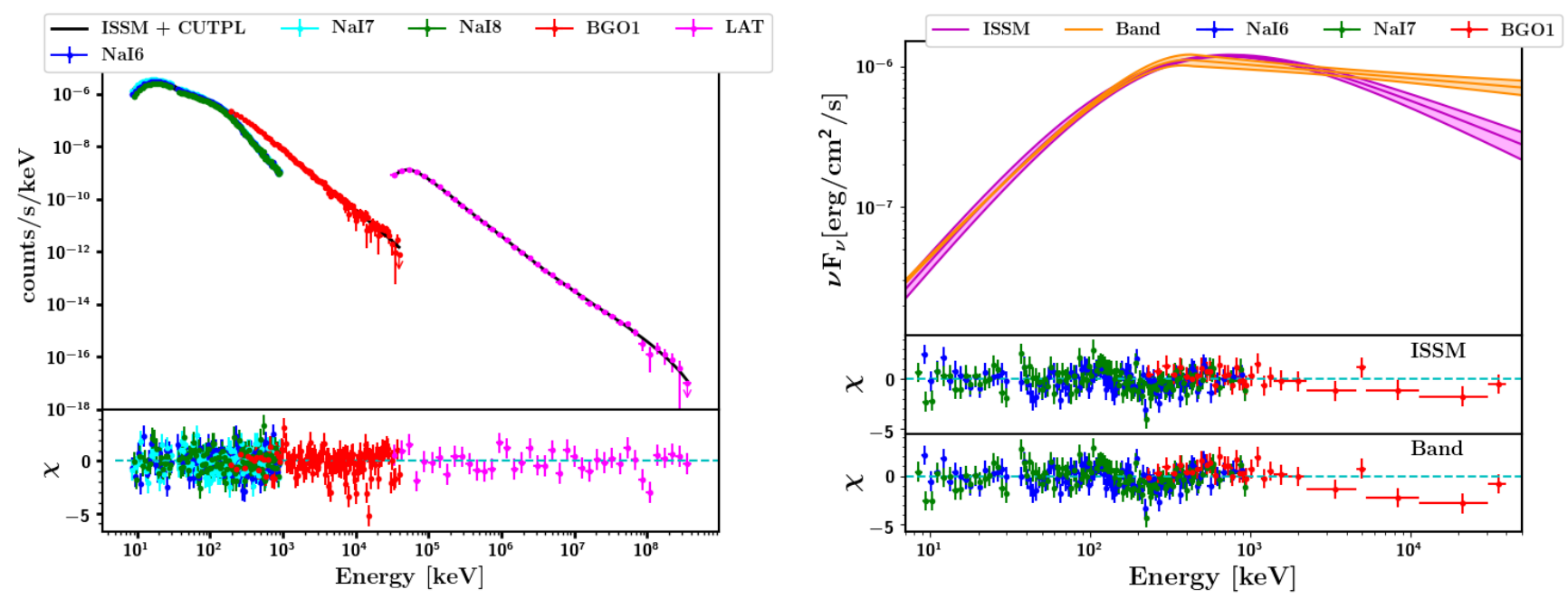

Fig. 4. Left: fit of the count spectrum of the synthetic burst GRB_B001 with the ISSM + CUTPL model in the time interval [1 s-3 s]. Right: fit of the ISSM and Band models to the GRB 150403913 spectrum oberved by the GBM.

model in the two time intervals are reported in Table 3 and shown in the left panel of Fig. 4 for the time interval [ $1 s-3 s]$. The fit residuals and reduced $C_{\text {stat }}$ values clearly show the excellent quality of the fits and the ability of the ISSM + CUTPL model to reproduce the broadband shape of the synthetic spectra.

\section{Application to GBM bursts}

\subsection{GRB sample and data selection}

According to the results presented in Sect. 4, a large number of counts are required to distinguish the different spectral model based on their fit quality. For this reason, we selected a sample of bursts detected by the GBM with an energy fluence larger than $10^{-5} \mathrm{erg} \mathrm{cm}^{-2}$ (from 10 to $1000 \mathrm{keV}$ ), namely comparable to those of the GRB_B001 and GRB_B010 synthetic bursts. Like in Sect. 4, we first focused our study on the sub-MeV spectral component, discarding the bursts that have additional components at low or high energies. This includes the GRBs with a low-energy excess which has been interpreted as a possible thermal component (GRB 090424, GRB 090820 Tierney et al. 2013, GRB 090902B Abdo et al. 2009, GRB 090926A Guiriec et al. 2015, GRB 100724B Guiriec et al. 2011, GRB 110721 Axelsson et al. 2012), the GRBs with an extra high-energy power-law component (GRB 080916C Ackermann et al. 2013, GRB 090902B Abdo et al. 2009, 

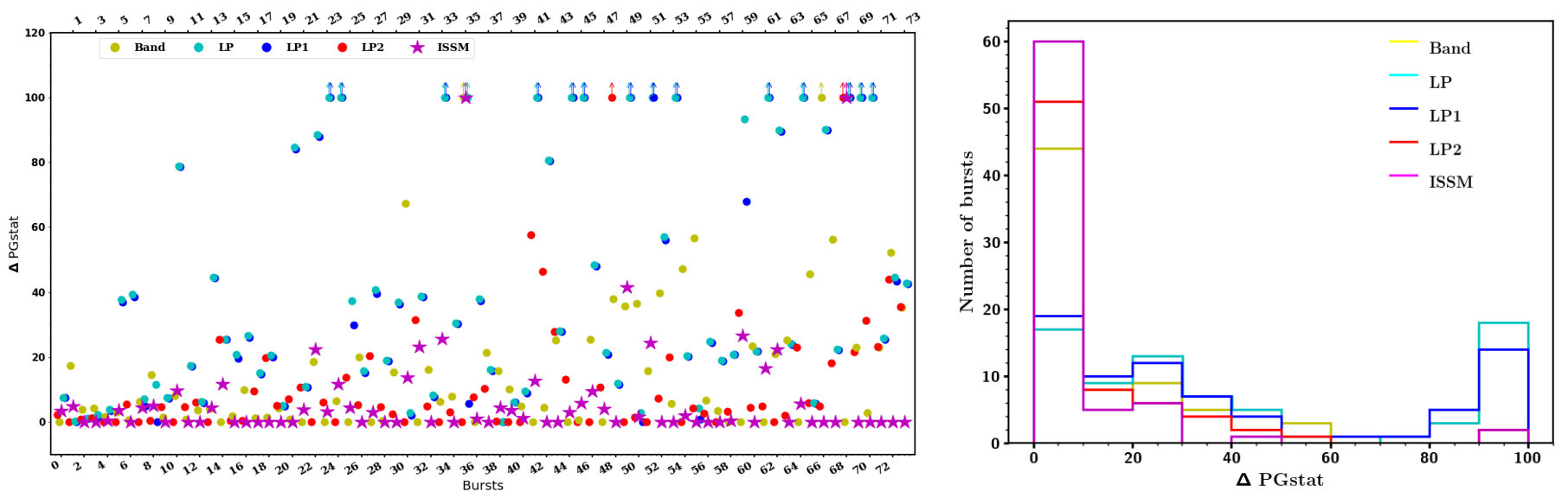

Fig. 5. Left: difference in $\mathrm{PG}_{\text {stat }}$ of the five models with respect to the model with the lowest $\mathrm{PG}_{\text {stat }}$, for every GRB displayed with increasing $\mathrm{SNR}$ (increasing from the left to the right). For each GRB, the five model markers are displayed within two vertical (red and blue) lines. By definition, the model with the lowest $\mathrm{PG}_{\text {stat }}$ is always placed on the zero horizontal line. Lower limits at the top of the figure stand for models with a $\Delta \mathrm{PG}_{\text {stat }}$ larger than 100. Right: distribution of $\Delta \mathrm{PG}_{\text {stat }}$ for the five models.

GRB 090926A Ackermann et al. 2011), or those with a strong spectral evolution (GRB 081215A Tierney et al. 2013). The bursts whose spectra are best fit by a simple power law in the GBM spectral catalog ${ }^{5}$ (Gruber et al. 2014) were also excluded. Beside these 15 GRBs, we eliminated the bursts that had been seen by $\mathrm{NaI}$ detectors with a separation angle between the detector axis and the source larger than $60^{\circ}$. As a result, we selected 74 GBM GRBs in the first eight observing years, which are listed in Table A.4. More than half of them (41) are best fit by the Band function in the GBM spectral catalog (Gruber et al. 2014). Another fair fraction of bursts from this catalog (24) are best fit by a power law with an exponential cutoff. This model is a special case of the Band function that is obtained for a very steep high-energy index (i.e., $\beta$ tends to $-\infty$ and $E_{\mathrm{b}}$ to $+\infty$ in Eq. (2)). The remaining nine GRBs were found to be best fit by a smoothly broken power law by Gruber et al. (2014), which is characterized by a flexible SED width around its peak energy. The data are loaded from the FSSC GBM data ${ }^{6}$ using the gtburst tool $^{7}$. The spectral analyses where performed during the T90 defined in the GBM catalog (Gruber et al. 2014). For each GRB of the sample, we selected one BGO detector with a separation angle less than $90^{\circ}$ and a maximum of three NaI detectors that had seen the burst with a separation angle less than $60^{\circ}$.

\subsection{Model comparison}

We performed a spectral analysis of the 74 selected GRBs with the XSPEC software and for the five spectral models; Band, LP, LP1, LP2 and ISSM. The reference energies $E_{0}$ in Eqs. (4), (6), (7) and $E_{\mathrm{r}}$ in Eq. (13) of the LP, LP1, LP2 and ISSM functions, were fixed to $500 \mathrm{keV}$. We used the "Poisson-Gauss" fit statis$\mathrm{tic}^{8}$ ( $\mathrm{PG}_{\text {stat }}$ hereafter), which is suitable for GRB spectral analysis, where the observed data counts are Poisson distributed in the energy channels, while background counts were estimated beforehand from pre- and post-burst data and are assumed to

\footnotetext{
5 https://heasarc.gsfc.nasa.gov/W3Browse/fermi/ fermigbrst.html

6 https://fermi.gsfc.nasa.gov/ssc/data/access/gbm/

7 https://fermi.gsfc.nasa.gov/ssc/data/analysis/ scitools/gtburst.html

8 See https://heasarc.gsfc.nasa.gov/xanadu/xspec/ manual/XSappendixStatistics.html
}

follow a Gaussian distribution. The case of GRB 150403913 is shown in the right panel of Fig. 4 for the ISSM and Band fits. The left panel of Fig. 5 shows the increase of $\mathrm{PG}_{\text {stat }}$ of the five models with respect to the model which has the lowest $\mathrm{PG}_{\text {stat }}$ ("reference model" hereafter). In this panel, the GRBs are displayed in order of increasing signal-to-noise ratio (SNR), which is defined for each GRB as:

$$
\mathrm{SNR}=\sum_{i=1}^{N}\left(c_{\mathrm{i}}-b_{\mathrm{i}}\right) / \sqrt{\sum_{i=1}^{N} b_{\mathrm{i}}}
$$

where $c_{\mathrm{i}}\left(b_{\mathrm{i}}\right)$ are the total (background) counts recorded by the $N$ NaI detectors that detected the burst. The right panel of Fig. 5 shows the resulting distribution of $\Delta \mathrm{PG}_{\text {stat }}$ for the five models. The ISSM function has the lowest $\mathrm{PG}_{\text {stat }}$, namely, it is the reference model, for half of the GRBs in Fig. 5. Since the ISSM function shows the lowest value of $\mathrm{PG}_{\text {stat }}$ in half of the cases, it is taken as a reference (level 0 on the bottom of Fig. 5) and the other models are displayed accordingly. The first GRBs with the minimum SNR values in this figure have comparable $\mathrm{PG}_{\text {stat }}$ values for the five spectral models and the $\Delta \mathrm{PG}_{\text {stat }}$ increases with SNR as expected, since the models can be more easily distinguished from each other with a larger event statistics.

To compare the fit models with each other, we used the $\Delta \mathrm{PG}_{\text {stat }}$ as a likelihood ratio test (Neyman \& Pearson 1928). In case of nested models, where the model parameterization in the null hypothesis is a special case of that in the alternative hypothesis, the $\Delta \mathrm{PG}_{\text {stat }}$ is expected to follow a $\chi^{2}$ distribution with $k$ degrees of freedom in the large sample limit, where $k$ is the number of additional parameters between the two models (Wilks 1938). Since several of the models that we considered are not nested, and because the large sample limit is not reached in all energy channels of the GRBs in our sample, one should compute the $\triangle \mathrm{PG}_{\text {stat }}$ probability density function for each GRB and each pair of models by simulating a large number of spectra. Given the vast number of cases, we focused on the Band and ISSM functions, in the two cases of a low or a medium value of the SNR. We performed Monte Carlo simulations for two cases in our sample, GRB 100910A (SNR = 141) and GRB 110921A (SNR $=249)$, considering the Bandfunction as the null hypothesis. We used the XSPEC software to simulate $10^{5}$ Band spectra for the duration of each GRB, using the DRM and background files of the GBM detectors that have seen the burst with the 

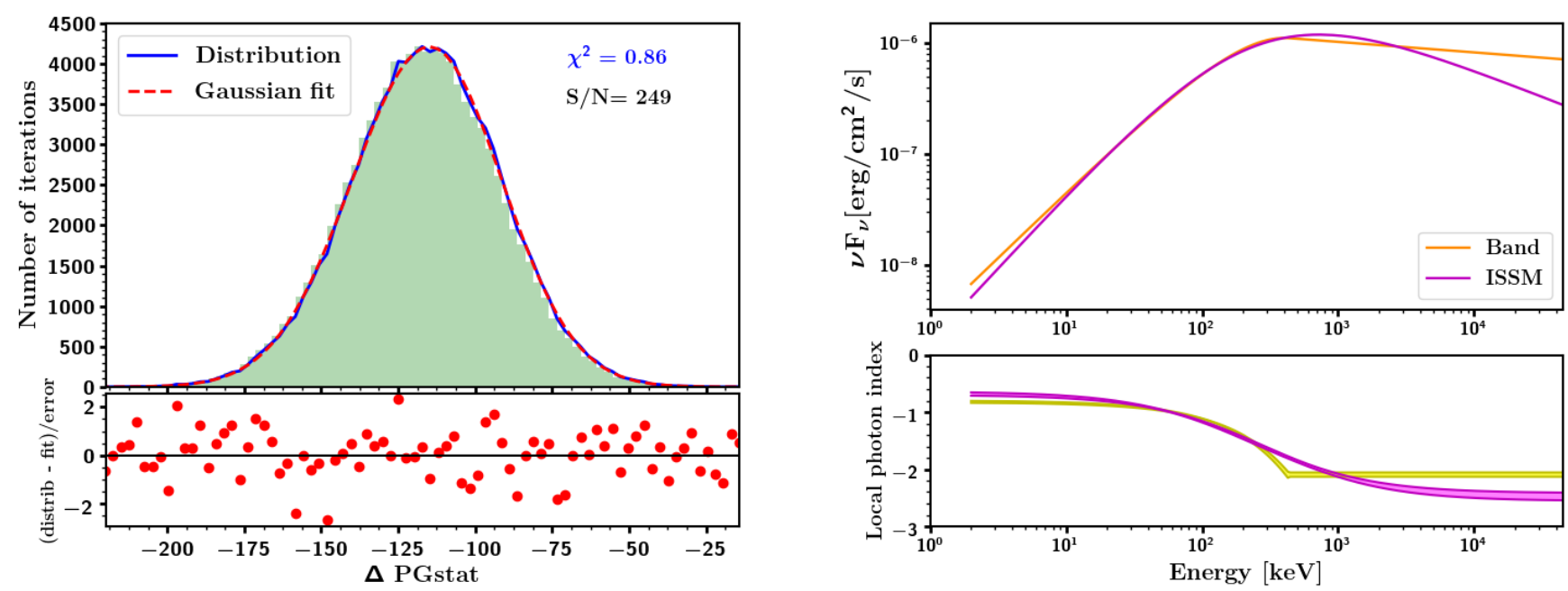

Fig. 6. Left: fit of an asymmetric Gaussian function to the distribution of $\Delta \mathrm{PG}_{\mathrm{stat}}$ between the Band and the ISSM function for the medium SNR case. Bottom panel: ratio of the difference between the histogram and its fit over the error ( $\sqrt{N}$ in each bin). Right: spectral energy distribution and local photon index of a representative burst (GRB 150403913) with the Band and ISSM functions.

best viewing angle. All the simulated spectra were then fitted with the Band and ISSM functions. The resulting distribution of $\Delta \mathrm{PG}_{\text {stat }}=\mathrm{PG}_{\text {stat,Band }}-\mathrm{PG}_{\text {stat,ISSM }}$ for GRB 110921A is shown in the left panel of Fig. 6. The fit of this distribution with an asymmetric Gaussian function and its extrapolation allowed us to compute the $\Delta \mathrm{PG}_{\text {stat }}$ limit beyond which the probability that a statistical fluctuation yields a better fit with the ISSM function than with the Band function is smaller than $10^{-6}$ (approximately 5 Gaussian standard deviations). The limit was found to be $\Delta \mathrm{PG}_{\text {stat }}=20$ for a low SNR and 3 for a medium SNR, beyond which the null hypothesis (i.e., the Band function) must be rejected. Because it was complicated and time consuming to determine a limit for each GRB and each pair of models, we adopted a common limit of $\Delta \mathrm{PG}_{\text {stat }}=10$ in all situations.

As a result, this study revealed that the ISSM model is the reference model for 36 GRBs, 19 of which are equivalently fit by the Band function. On the contrary, the Band function has the lowest $\mathrm{PG}_{\text {stat }}$ value for $16 \mathrm{GRBs}, 10$ of which are equivalently fit by the ISSM function. Concerning the other three models, only the LP2 showed good performance. It is the reference model for 18 GRBs, and globally as good as the Band model, though with one more parameter. All in all, the ISSM function is a good spectral model for $81 \%(60 / 74)$ of the GRBs in our sample, namely in these cases it is the reference model or it is close enough to it in terms of $\mathrm{PG}_{\text {stat }}$. The Band function was found to be a good spectral model for a smaller fraction $(59 \%)$ of the GRB sample (44/74), similar to the LP2 function (65\%), versus only 20\% for the LP1 and LP functions. It must be noted that these performances would improve for more common and less fluent bursts with lower signal-to-noise ratios.

\subsection{Band and ISSM spectral parameters}

In this section we compare the spectral parameters of the Band and ISSM functions. The left panel of Fig. 7 shows the SED peak energies obtained with the two models. The $E_{\mathrm{p}}$ values of the ISSM function are found to be systematically larger than the values obtained with the Band function. The low-energy index $\alpha$ is an asymptotic value that is rarely reached by the local photon index within the energy range of any burst-observing instrument. For this reason, Preece et al. (1998) defined an effective lowenergy index at the CGRO/BATSE detector lower limit $(25 \mathrm{keV})$.
In order to find the energy limit $\left(E_{\mathrm{lim}}\right)$ at which the local photon index $\Gamma(E)$ approaches the asymptotic value $\alpha$ within its error $\delta \alpha$, we solved the equation $\Gamma\left(E_{\text {lim }}\right)=\alpha-\delta \alpha$ using the definition of the local photon index of the Band and ISSM functions in Eqs. (3) and (15), respectively. The $E_{\text {lim }}$ energies of the two functions are expressed as:

$$
\begin{aligned}
& E_{\text {lim,Band }}=\frac{\delta \alpha}{2+\alpha} E_{\mathrm{p}} \\
& E_{\text {lim,ISSM }}=\frac{\delta \alpha(2+\beta)}{(2+\alpha)(\beta-\alpha+\delta \alpha)} E_{\mathrm{p}} .
\end{aligned}
$$

These quantities are displayed with respect to $E_{\mathrm{p}}$ in the right panel of Fig. 7. For the vast majority of the GRBs in our sample, the $E_{\text {lim }}$ values fall below the GBM energy range. We thus defined $\alpha_{10}$ as the local photon index at $10 \mathrm{keV}$, namely right above the low-energy detection limit of the GBM. The left panel of Fig. 8 compares the $\alpha_{10}$ index to the $\alpha$ asymptotic index for both the Band and ISSM functions. While the $\alpha$ indices of the ISSM function are larger than those of the Band function, the $\alpha_{10}$ indices of the ISSM function are only slightly larger. The values of $\alpha_{10}$ also appear less scattered than those of $\alpha$. More interestingly, the fraction of GRBs that are fit with the ISSM function and whose index is harder than the synchrotron slow-cooling limit $(-2 / 3)$ decreases from $35 \%$ ( $\alpha$ asymptotic index) to $26 \%\left(\alpha_{10}\right)$. This fraction decreases from $19 \%$ to $12 \%$ for the Band function. As shown in the right panel of Fig. 8, which displays the $\alpha$ and $\alpha_{10}$ distributions for both models, the weighted mean index of the ISSM (Band) function indeed decreased from $\langle\alpha\rangle=-0.75$ $(-0.88)$ to $\left\langle\alpha_{10}\right\rangle=-0.97(-1.03)$.

Similarly, the $\beta$ parameter of the ISSM function is an asymptotic value at high energy, which may not be reached by the local photon index within the GBM energy range. Therefore, we defined $\beta_{\mathrm{b}}$ as the photon index at the break energy $E_{\mathrm{b}}$ of the Band function (Eq. (2)). By definition, $\beta_{\mathrm{b}}$ is equal to $\beta$ for the Band function, while is it harder than $\beta$ for the ISSM function owing to its continuous curvature. The $\beta_{\mathrm{b}}$ index of the ISSM function was also found to be systematically harder than that of the Band function, namely $\beta_{\text {ISSM }}<\beta_{\text {Band }}<\beta_{\text {b,ISSM. }}$. As a result, GRB spectra appear slightly wider around their peak energy when fit with the ISSM function rather than with the Band function, but narrower when observed over a wider energy range. This is illustrated in 

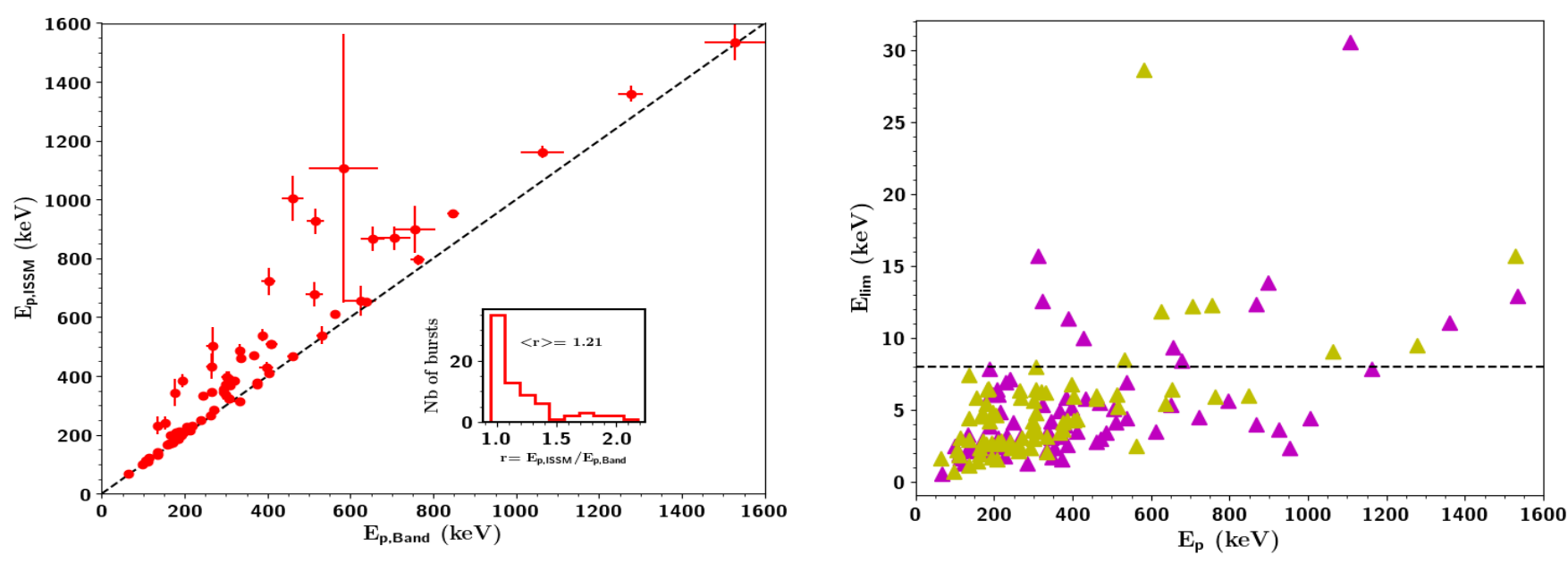

Fig. 7. Left: comparison of the $E_{\mathrm{p}}$ parameter between the Band and ISSM functions. The dashed line is the equality line. The inset shows the ratio between the $E_{\mathrm{p}}$ values obtained by ISSM and Band functions. Right: limit energies $E_{\mathrm{lim}}$ of the ISSM and Band functions compared with their peak energies $E_{\mathrm{p}}$. The horizontal dashed line represents the lower limit of the GBM energy range.
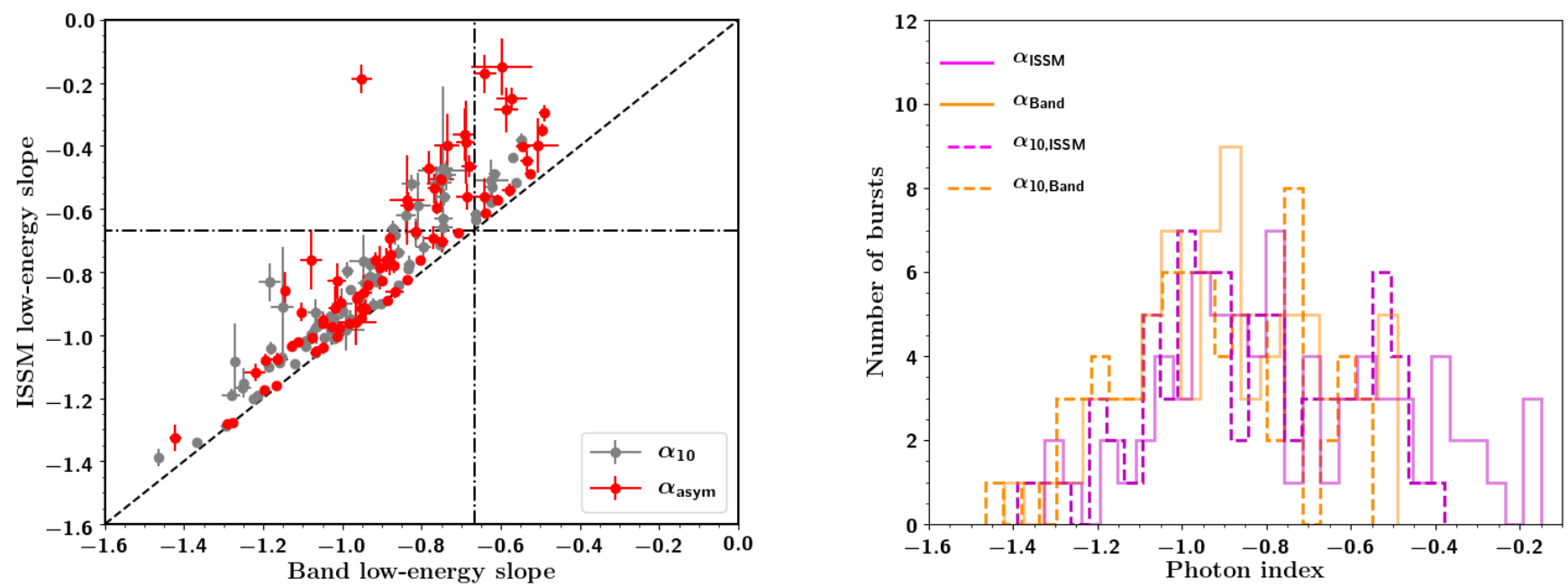

Fig. 8. Left: comparison of the asymptotic $\alpha$ and the local photon index $\alpha_{10}$ at $10 \mathrm{keV}$ between the Band and ISSM functions. The gray dashed line denotes equality. The dashed-dotted horizontal and vertical lines indicate the upper limit $(-2 / 3)$ of the low-energy spectral index for synchrotron emission in the slow cooling regime. Right: distributions of the $\alpha$ and $\alpha_{10}$ parameters of the Band and ISSM functions.

the right panel of Fig. 6, for the case of GRB 150403913, which is best fit by the ISSM model.

\subsection{Band and ISSM spectral sharpness}

We investigated how the sharpness of the Band and ISSM fitted spectra varies quantitatively with the photon indices. Following the methodology described in Sect. 4, a set of $10^{3}$ spectra was simulated for each GRB using its fit parameters and their covariance matrix. The spectral sharpness angles of the GRB sample are presented in the left panel of Fig. 9. Similarly to the synthetic bursts analyzed in Sect. 4, the ISSM spectra are slightly wider than the Band spectra. As expected, the spectral sharpness angle was found to be independent of the peak energy, and to depend strongly on the photon indices. As shown in the right panel of Fig. 9, the spectral sharpness angle decreases with increasing $\alpha_{10}$ and/or with decreasing $\beta$. The spectral sharpness angles of the GRBs in our sample are similar to those obtained by Yu et al. (2015; see figures therein, e.g., the blue solid curve in the left panel of Fig. 7), ranging from $\sim 115^{\circ}$ to $\sim 140^{\circ}$ in both analyses, except one with a $152^{\circ}$ angle with the ISSMfunction.
More importantly, the spectral sharpness angle of the synthetic bursts fit by the ISSM function is $149^{\circ}$ (see Table 2), which is larger than for any GRB in our sample using the same fitting model. This essentially results from the difference in the lowenergy spectral index, $\alpha \simeq-1.2$, which is softer than for most of the analyzed GRBs (see the left panel of Fig. 8). Besides, the value of the high-energy index of the synthetic burst, $\beta \simeq-2.3$, is close to the higher bound of the sample distribution as shown in Fig. 10. Possible ways to improve the agreement between the synthetic and observed bursts are discussed in Sect. 7.

\section{Application to GRB 090926A}

The prompt light curve of GRB 090926A shows a short and bright spike at $10 \mathrm{~s}$ post-trigger which was detected from $\mathrm{keV}$ to $\mathrm{GeV}$ energies by the Fermi instruments (Ackermann et al. 2011). This spike coincides with the emergence of a hard powerlaw spectral component that is attenuated at the highest energies. In Y17, we performed a dedicated analysis of the broadband prompt emission spectrum of GRB 090926A by combining the GBM data with the LAT Pass 8 data above $30 \mathrm{MeV}$. This analysis 

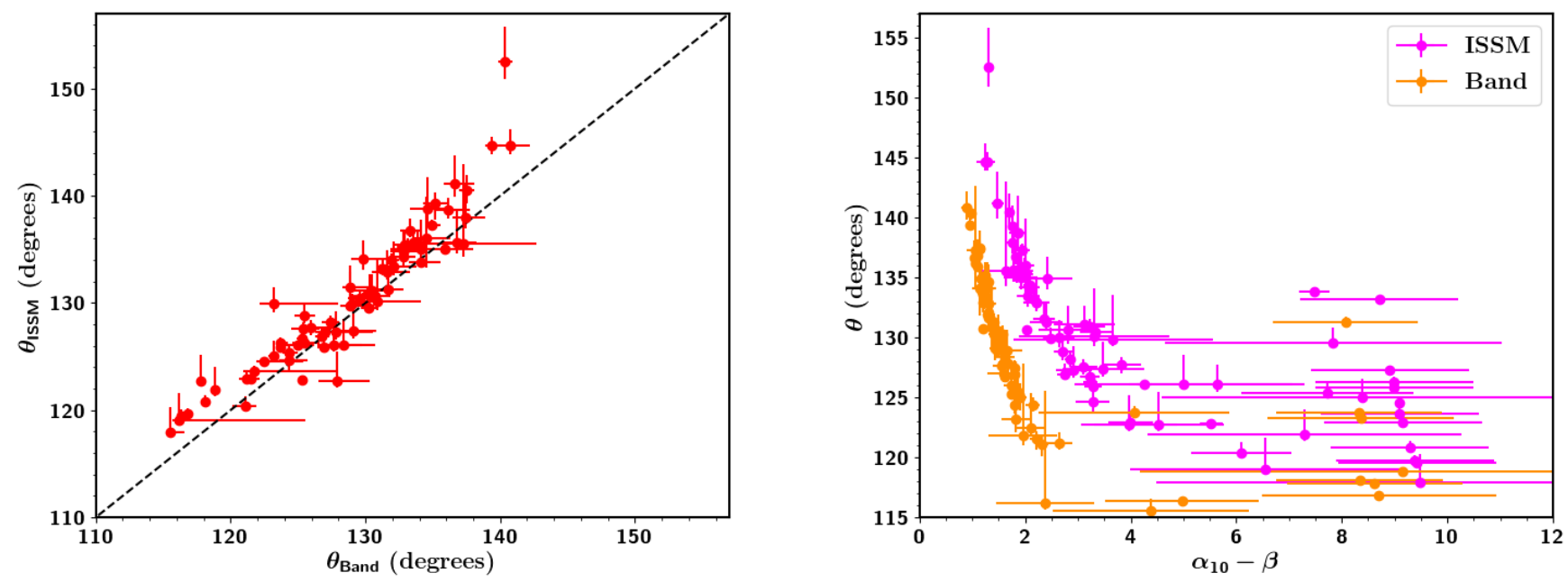

Fig. 9. Left: spectral sharpness angles of the ISSM fits versus the angles of the Band fits to the GRB spectra. Right: spectral sharpness angle as function of the difference between the $\alpha_{10}$ and $\beta$ parameters for the Band and ISSM functions.
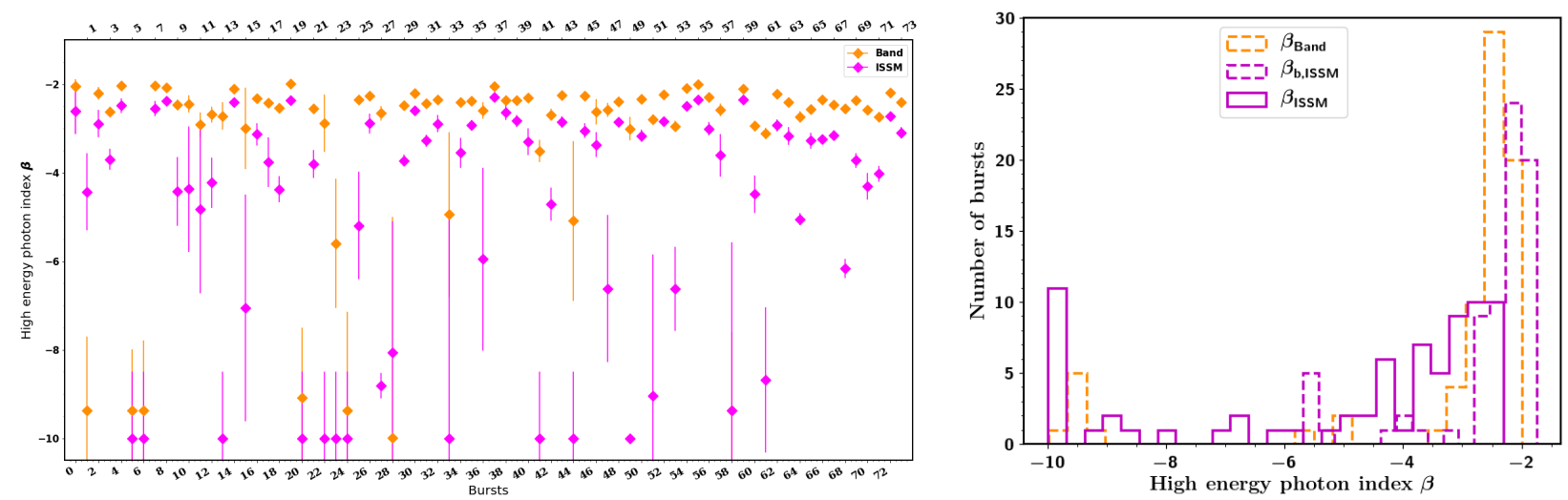

Fig. 10. Left: distribution of high-energy index $\beta$ of the Band and ISSM functions. The lowest limit value used for $\beta$ was fixed to -10 . Right: distributions of $\beta$ parameter of the Band and ISSM functions.

used a Band + CUTBPL fitting function and showed that the spectral break energy increases with time, and that the entire prompt emission of this burst, namely the emission that is observed from $\mathrm{keV}$ to $\mathrm{GeV}$ energies by the GBM and the LAT during the GRB duration in the 50-300 keV energy band, can be interpreted as the result of synchrotron emission of shock-accelerated electrons in the keV-MeV domain, with an inverse Compton spectral component at higher energies. The latter component was fit by the CUTBPL function instead of the CUTPL function to avoid any unrealistic contribution to the observed flux in the GBM lowenergy range. As a result, the low-energy index $\alpha$ of the Band spectral component was found to be close to -0.9 , which is in agreement with the theoretical index $(\sim-1)$ of the fast-cooling synchrotron spectrum that is expected in the presence of inverse Compton scatterings in the Klein-Nishina regime (BD14).

Going further, we revisited the spectral analysis of GRB 090926A and compared the Band + CUTBPL model to the ISSM + CUTBPL model. This analysis was performed with the XSPEC software for the time intervals $c(0.98 \mathrm{~s}$ to $10.5 \mathrm{~s})$ and $d(10.5 \mathrm{~s}$ to $21.5 \mathrm{~s})$ where the high-energy break is detected. Following Y17, we fixed the parameters $\gamma_{0}$ and the break energy $E_{\mathrm{b}}$ of Eq. (9) to +4 and $200 \mathrm{keV}$, respectively. Like in Y17, the reference energy $E_{0}$ was fixed to $10 \mathrm{MeV}$ and $100 \mathrm{MeV}$ for the time intervals $c$ and $d$, respectively. The results of this analysis are presented in Fig. 11 and summarized in Table 4. As can be seen in all panels of this figure and from the $\mathrm{PG}_{\text {stat }}$ fit statistics, both the Band + CUTBPL and ISSM + CUTBPL models reproduce adequately the GRB spectrum, especially in the time interval $c$ (top panels). The low-energy indices $\alpha$ of the Band and ISSM spectral components are equal within statistical errors, and close to -1 and -0.9 for the time intervals $c$ and $d$, respectively. Again, these values perfectly agree with the predictions of BD14. All other spectral parameters are also equivalent between both models, except the high-energy index $\beta$ of the keV-MeV spectral component, which is not well constrained using the ISSM function. Since the ISSM flux decreases more rapidly than that of the Band function beyond the SED peak energy, this likely results from the lack of photon statistics in the SED dip at a few $\mathrm{MeV}$, between the GBM and LAT energy domains.

\section{Discussion}

Our analysis of a sample of 74 GRBs that are bright and fluent in the GBM showed that the ISSM function adequately reproduces most $(81 \%)$ of the keV-MeV prompt emission spectra, while the Band phenomenological function is suitable for a smaller fraction $(59 \%)$. We observed noticeable differences between the spectra fit with these two functions. The peak energies $E_{\mathrm{p}}$ of the spectra that are reconstructed using the ISSM function are somewhat higher than those of the spectra resulting from the Band 

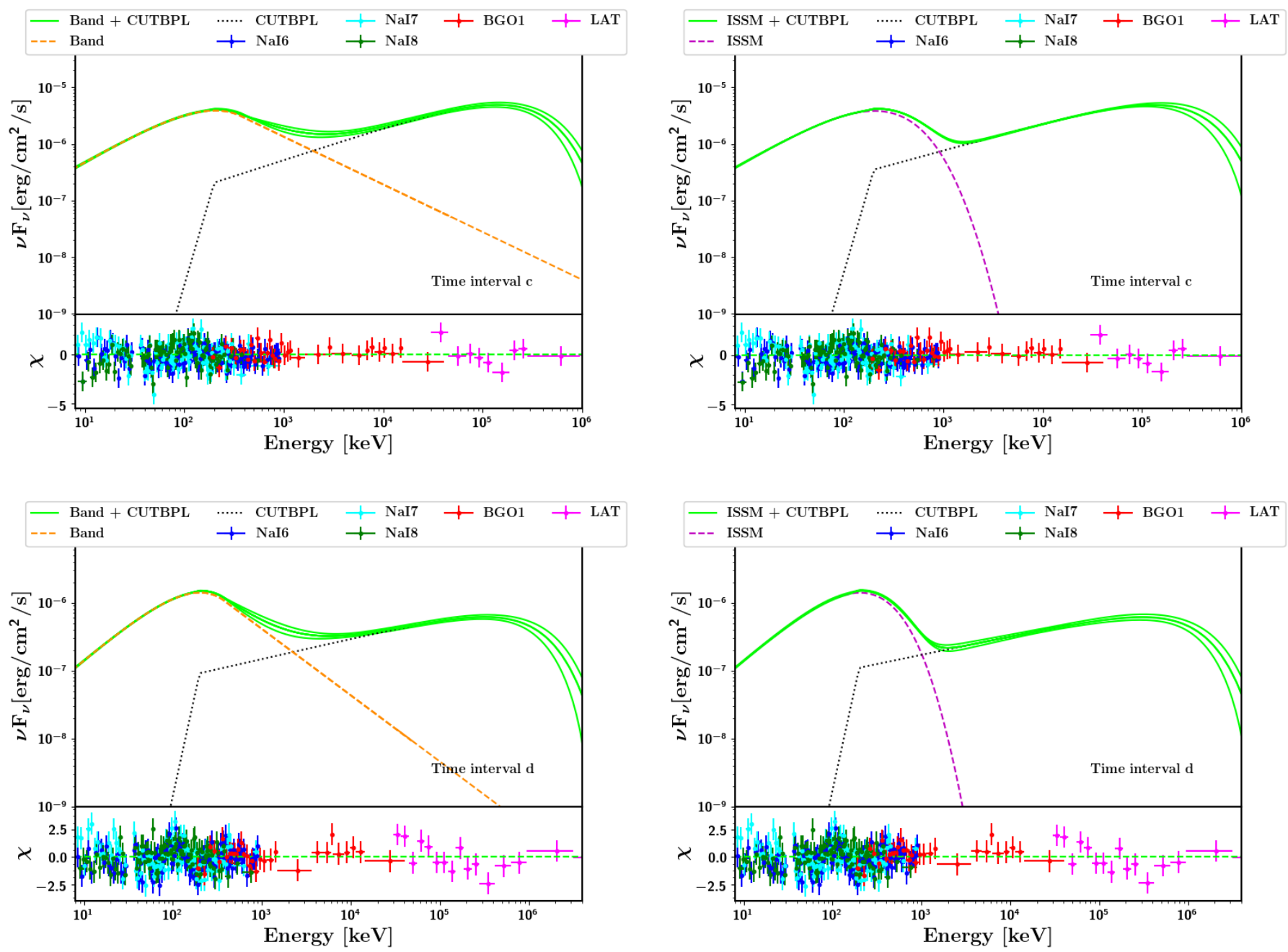

Fig. 11. Spectral energy distributions of GRB 090926A in time intervals $c$ (top panels) and $d$ (lower panels) from the joint GBM/LAT analysis using LAT Pass 8 above $30 \mathrm{MeV}$. The solid curve represents the Band + CUTBPL model (left column) and the ISSM + CUTBPL model (right column), within a $68 \%$ confidence level contour derived from the errors on the fit parameters. The reference energy of the CUTBPL was fixed to $10 \mathrm{MeV}$ like in Y17.

Table 4. Results of the Band + CUTPL and ISSM + CUTBPL fits to GBM/LAT data in the time intervals $c$ and $d$ of GRB 090926A.

\begin{tabular}{|c|c|c|c|}
\hline Time intervals & Parameters & Band + CUTPL & ISSM + CUTBPL \\
\hline$[0.98 \mathrm{~s}-10.5 \mathrm{~s}]$ & $\begin{array}{l}A_{\text {Band/ISSM }}\left(\mathrm{keV}^{-1} \mathrm{~cm}^{-2} \mathrm{~s}^{-1}\right) \\
E_{\mathrm{p}}(\mathrm{keV}) \\
\alpha \\
\beta \\
A_{\text {CUTBPL }}\left(\times 10^{4} \mathrm{keV}^{-1} \mathrm{~cm}^{-2} \mathrm{~s}^{-1}\right) \\
\lambda \\
E_{\mathrm{f}}(\mathrm{GeV}) \\
\mathrm{PG}_{\text {stat }} / \text { d.o.f. }\end{array}$ & $\begin{array}{c}0.33_{-0.01}^{+0.02} \\
203_{-7}^{+7} \\
-0.98_{-0.03}^{+0.03} \\
-2.8_{-0.3}^{+0.2} \\
2.04_{-0.89}^{+1.37} \\
-1.43_{-0.11}^{+0.10} \\
0.25_{-0.05}^{+0.07} \\
577 / 510\end{array}$ & $\begin{array}{c}580_{-5}^{+5}\left(\times 10^{-5}\right) \\
203_{-1}^{+1} \\
-0.97_{-0.02}^{+0.02} \\
-14.0_{-1.0}^{+0.7} \\
3.46_{-0.32}^{+0.22} \\
-1.52_{-0.02}^{+0.02} \\
0.27_{-0.05}^{+0.07} \\
582 / 510\end{array}$ \\
\hline$[10.5 \mathrm{~s}-21.5 \mathrm{~s}]$ & $\begin{array}{l}A_{\text {Band/ISSM }}\left(\mathrm{keV}^{-1} \mathrm{~cm}^{-2} \mathrm{~s}^{-1}\right) \\
E_{\mathrm{p}}(\mathrm{keV}) \\
\alpha \\
\beta \\
A_{\text {CUTBPL }}\left(\times 10^{7} \mathrm{keV}^{-1} \mathrm{~cm}^{-2} \mathrm{~s}^{-1}\right) \\
\lambda \\
E_{\mathrm{f}}(\mathrm{GeV}) \\
\mathrm{PG}_{\text {stat }} / \text { d.o.f. }\end{array}$ & $\begin{array}{c}0.123_{-0.003}^{+0.003} \\
201_{-5}^{+5} \\
-0.88_{-0.02}^{+0.02} \\
-3.0_{-0.3}^{+0.1} \\
8.89_{-2.72}^{+2.92} \\
-1.70_{-0.05}^{+0.06} \\
1.08_{-0.25}^{+0.31} \\
714 / 510\end{array}$ & $\begin{array}{c}203_{-11}^{+16}\left(\times 10^{-5}\right) \\
204_{-5}^{+7} \\
-0.86_{-0.02}^{+0.02} \\
-14_{-1}^{+9} \\
10.74_{-2.93}^{+1.65} \\
-1.73_{-0.04}^{+0.05} \\
1.12_{-0.25}^{+0.32} \\
717 / 510\end{array}$ \\
\hline
\end{tabular}


function fits. In addition, the ISSM fit spectra are globally narrower than the Band fit spectra, yet they appear slightly wider close to $E_{\mathrm{p}}$. This results in slightly larger sharpness angles for the ISSM fit spectra, which were also observed from fits of the synthetic spectrum.

Although the shape of the ISSM function seems adequate to reproduce the spectral curvature of the GBM bright bursts, the spectral sharpness angle in this sample is always smaller than the sharpness angle of the synthetic spectra that were used to build this fitting function. Since the spectral sharpness angle scales almost linearly with $\beta-\alpha$ (right panel of Fig. 9), it is worth investigating possible ways to improve the agreement between the data and the physical model. Firstly, the high-energy photon index $\beta$ in the model is strongly related to the slope of the electron power-law energy distribution $p$, as $\beta=-(p / 2+1)$ in the synchrotron fast-cooling regime. While $p=2.7$ and thus $\beta=-2.3$ for the synthetic bursts, larger values of $p$ up to 2.9 could be considered (BD14), owing to the theoretical uncertainties on the energy distribution of accelerated electrons in midlyrelativistic shocks. However, the expected change in the value of $\beta$ would not entirely account for the sharpness discrepancy with observed spectra. Moreover, many of the observed values of $\beta$ are larger for the bursts with well-measured spectral parameters as shown in Fig. 10. In this figure, softer high-energy indices appear with larger uncertainties and might be underestimated due to insufficient photon statistics above the peak energy. This suggests that a better spectral coverage at $\mathrm{MeV}$ energies could result in harder values of $\beta$ and in larger sharpness angles for this fraction of the burst sample, making the entire sample compatible with the physical model.

Secondly, the low-energy photon index $\alpha$ of the synthetic spectra is close to -1.2. As shown in Fig. 9, an increase of 0.5 in this theoretical slope, with a condition that the high-energy slope does not increase, would be enough to make the synthetic spectra compatible with the GBM sample in terms of spectral sharpness. As a matter of fact, harder values of $\alpha$ are expected from internal shock synchrotron models in the so-called marginally fastcooling regime (Daigne et al. 2011; Beniamini \& Piran 2013), where the impact of adiabatic losses on the electron energy distribution is not negligible anymore when compared to the effect of radiative losses. In this regime, specific configurations of the jet such as low-contrast internal shocks can lead to $\alpha$ values as hard as -2/3 (Daigne et al. 2011). Hardening the low-energy part of the synchrotron spectrum could also be obtained by accounting for the decay of the magnetic field behind the shock (Pe'er \& Zhang 2006; Derishev 2007). In such a configuration, the most energetic electrons would indeed explore a small region where the magnetic field has not decreased yet, while the less energetic electrons would see a less intense magnetic field on average. Therefore, such a magnetic field decay appears as a natural possibility to reach the marginally fast cooling regime without any need for a fine-tuning the microphysical parameters (Daigne \& Bošnjak, in prep.). Indeed, as the magnetic field decreases, the critical Lorentz factor of electrons for slow cooling $\gamma_{\mathrm{c}}$ increases.

Preliminary results show that a steep asymptotic slope $\alpha$ close to $-2 / 3$ is obtained. This effect of a magnetic field decay is shown in the right panel of Fig. 2 where the value of the ISSM low-energy slope $\alpha=b / c-a$ varies between -1.5 (case A with a constant magnetic field) and -0.8 (case B with a magnetic field decay for the spectrum measured at the peak of the light curve). This illustrates that a more realistic modeling of the microphysics in the acceleration and emission regions should be investigated to reach a full agreement between the synthetic and observed spectra. Ultimately one would like to use the spectral fits to infer the physical parameters of the model such as the evolution of the injected power or the distribution of the Lorentz factor in the flow. In practice, analysis of the spectra only provides values for the four parameters: $A_{\mathrm{ISSM}}, \alpha, \beta$ and $E_{\mathrm{p}}$. They partially constrain the shock physics and radiative mechanism as discussed above for $\alpha$ and $\beta$. The peak energy $E_{\mathrm{p}}$ depends on a combination of the ejecta physical parameters and shock microphysics. It will therefore be difficult to decipher from the evolution of $E_{\mathrm{p}}$ the form of the Lorentz factor or/and injected power distributions even if some general trends can probably be obtained. This will require a dedicated study.

\section{Conclusions}

The physical origin of GRB prompt emission remains elusive despite decades of observations. Characterizing the prompt emission spectra has been often performed using phenomenological parameterizations with little physical grounds, such as the Band function. However, the advance of instrument spectral coverage and the improved data quality provided by current missions such as the Fermi observatory now offer the possibility to confront observations to theoretical models in detail. In this work, we used the internal shock model developed by BD14 to produce synthetic GRBs (see also Bošnjak et al. 2009; Daigne et al. 2011), and we folded their spectra with the response of the Fermi GBM and LAT. The synthetic spectra obtained from these simulations in the keV-MeV domain, where the synchrotron emission is dominant, were used to build a new GRB spectral fitting function called ISSM, which has the same number of parameters as the Band function. We used the ISSM function to fit the prompt emission spectra for a sample of 74 GBM fluent bursts, which improved the fit quality as compared to the phenomenological Band function in a sizeable number of cases. In addition, we combined the ISSM function with a CUTBPL spectral component to fit the GRB 090926A broadband spectrum with some success. This work was motivated by a previous study of this burst that suggested an internal origin of the $\mathrm{keV}$ to $\mathrm{GeV}$ emission observed during the prompt phase (Y17). In this framework, our interpretation of both spectral components as being from synchrotron and inverse Compton emissions would greatly benefit from a more realistic parameterization of the high-energy component based on the synthetic spectra, especially in the overlapping region at $\mathrm{MeV}$ energies.

The analysis of the GBM sample of 74 bursts showed noticeable differences between the ISSM and Band fits. Peak energies and spectral sharpness angles that are obtained from the ISSM fits are slightly larger than those from the Band fits. This result can be attributed to the continuous curvature of the ISSM function. This curvature reflects the time evolution of the electron and photon energy distributions within the analysed time intervals, which lasts longer than the typical dynamical timescales in the physical model. While observed spectra can be well fitted by the ISSM physical function, they appear narrower than the synthetic spectra, essentially because of a theoretical lowenergy photon index that differs significantly from the observed photon index $\alpha$. This problem clearly calls for improvements of the internal shock model and possible solutions have been identified. In particular, more sophisticated prescriptions for the jet physics should be investigated in the future, such as the marginally fast-cooling regime and the decay of the magnetic field behind the shocks. Inferring the parameters of the physical model from the fitted parameters of the ISSM function is not easy as their relation is complex. Actually, the physical arameters that best reproduce GRB prompt emission spectra should be 
rather explored by fitting the numerical model directly to the data in the future, without using the ISSM proxy function. On the experimental side, complementary multi-wavelength observations will be also performed by GRB-dedicated missions such as SVOM which will observe the complete time evolution of GRBs from possible precursors until the afterglow phase (Wei et al. 2016). SVOM will measure GRB prompt emission spectra down to $4 \mathrm{keV}$ thanks to its ECLAIRs coded-mask telescope, and up to the $\mathrm{MeV}$ range with its Gamma-Ray Monitor detector (Bernardini et al. 2017). This will provide more insight into the physical origin of GRB high-energy emission at early times.

Acknowledgements. The Fermi LAT Collaboration acknowledges generous ongoing support from a number of agencies and institutes that have supported both the development and the operation of the LAT as well as scientific data analysis. These include the National Aeronautics and Space Administration and the Department of Energy in the United States, the Commissariat à l'Energie Atomique and the Centre National de la Recherche Scientifique/Institut National de Physique Nucléaire et de Physique des Particules in France, the Agenzia Spaziale Italiana and the Istituto Nazionale di Fisica Nucleare in Italy, the Ministry of Education, Culture, Sports, Science and Technology (MEXT), High Energy Accelerator Research Organization (KEK) and Japan Aerospace Exploration Agency (JAXA) in Japan, and the K. A. Wallenberg Foundation, the Swedish Research Council and the Swedish National Space Board in Sweden. Additional support for science analysis during the operations phase is gratefully acknowledged from the Istituto Nazionale di Astrofisica in Italy and the Centre National d'Études Spatiales in France.

\section{References}

Abbott, B. P., Abbott, R., Abbott, T. D., et al. 2017, ApJ, 848, L13 Abdo, A. A., Ackermann, M., Ajello, M., et al. 2009, ApJ, 706, L138 Ackermann, M., Ajello, M., Asano, K., et al. 2011, APJ, 729, 114 Ackermann, M., Ajello, M., Asano, K., et al. 2013, ApJS, 209, 11 Ajello, M., Arimoto, M., Axelsson, M., et al. 2019, ApJ, 878, 52 Atwood, W. B., Abdo, A. A., Ackermann, M., et al. 2009, ApJ, 697, 1071

Atwood, W. B., Baldini, L., Bregeon, J., et al. 2013, ApJ, 774, 76 Axelsson, M., \& Borgonovo, L. 2015, MNRAS, 447, 3150 Axelsson, M., Baldini, L., Barbiellini, G., et al. 2012, ApJ, 757, L31 Band, D., Matteson, J., Ford, L., et al. 1993, ApJ, 413, 281 Beloborodov, A. M., \& Mészáros, P. 2017, Space Sci. Rev., 207, 87 Beniamini, P., \& Granot, J. 2016, MNRAS, 459, 3635

Beniamini, P., \& Piran, T. 2013, ApJ, 769, 69

Bernardini, M. G., Xie, F., Sizun, P., et al. 2017, Exp. Astron., 44, 113

Bloom, J. S., Kulkarni, S. R., Price, P. A., et al. 2002, ApJ, 572, L45
Bošnjak, Ž., \& Daigne, F. 2014, A\&A, 568, A45

Bošnjak, Ž., Daigne, F., \& Dubus, G. 2009, A\&A, 498, 677 Burgess, J. M. 2019, A\&A, 629, A69

Burgess, J. M., Ryde, F., \& Yu, H.-F. 2015, MNRAS, 451, 1511

Crider, A., Liang, E. P., Smith, I. A., et al. 1997, ApJ, 479, L39

Daigne, F., \& Mochkovitch, R. 1998, MNRAS, 296, 275

Daigne, F., Bošnjak, Ž., \& Dubus, G. 2011, A\&A, 526, A110

D’Avanzo, P. 2015, J. High Energy Astrophys., 7, 73

Derishev, E. V. 2007, Ap\&SS, 309, 157

Gehrels, N., Sarazin, C. L., O'Brien, P. T., et al. 2005, Nature, 437, 851

Ghisellini, G., Celotti, A., \& Lazzati, D. 2000, MNRAS, 313, L1

Giannios, D. 2008, A\&A, 480, 305

Gruber, D., Goldstein, A., Weller von Ahlefeld, V., et al. 2014, ApJS, 211, 12

Guiriec, S., Connaughton, V., Briggs, M. S., et al. 2011, ApJ, 727, L33

Guiriec, S., Kouveliotou, C., Daigne, F., et al. 2015, ApJ, 807, 148

Hjorth, J., Sollerman, J., Møller, P., et al. 2003, Nature, 423, 847

Hjorth, J., Sollerman, J., Gorosabel, J., et al. 2005, ApJ, 630, L117

Kawabata, K. S., Deng, J., Wang, L., et al. 2003, ApJ, 593, L19

Kobayashi, S., Piran, T., \& Sari, R. 1997, ApJ, 490, 92

Massaro, F., Grindlay, J. E., \& Paggi, A. 2010, ApJ, 714, L299

McKinney, J. C., \& Uzdensky, D. A. 2012, MNRAS, 419, 573

Nakar, E. 2007, Phys. Rep., 442, 166

Narayana Bhat, P., Meegan, C. A., von Kienlin, A., et al. 2016, ApJS, 223, 28

Neyman, J., \& Pearson, E. S. 1928, On the Use and Interpretation of Certain Test Criteria for Purposes of Statistical Inference, Part I (London: Cambridge University Press)

Oganesyan, G., Nava, L., Ghirlanda, G., \& Celotti, A. 2017, ApJ, 846, 137

Oganesyan, G., Nava, L., Ghirlanda, G., \& Celotti, A. 2018, A\&A, 616, A138

Oganesyan, G., Nava, L., Ghirlanda, G., Melandri, A., \& Celotti, A. 2019, A\&A, 628, A59

Pe'er, A., \& Zhang, B. 2006, ApJ, 653, 454

Preece, R. D., Briggs, M. S., Mallozzi, R. S., et al. 1998, ApJ, 506, L23

Ravasio, M. E., Oganesyan, G., Ghirlanda, G., et al. 2018, A\&A, 613, A16

Ravasio, M. E., Ghirlanda, G., Nava, L., \& Ghisellini, G. 2019, A\&A, 625, A60

Rees, M. J., \& Meszaros, P. 1994, ApJ, 430, L93

Ronchi, M., Fumagalli, F., Ravasio, M. E., et al. 2020, A\&A, 636, A55

Sironi, L., Petropoulou, M., \& Giannios, D. 2015, MNRAS, 450, 183

Stanek, K. Z., Matheson, T., Garnavich, P. M., et al. 2003, ApJ, 591, L17

Tierney, D., McBreen, S., Preece, R. D., et al. 2013, A\&A, 550, A102

Wei, J., Cordier, B., Antier, S., et al. 2016, The Deep and Transient Universe in the SVOM Era: New Challenges and Opportunities - Scientific Prospects of the SVOM Mission, Report on the Scientific Prospects of the SVOM Mission. Proceedings of the Workshop held from 11th to 15th April 2016

Wilks, S. S. 1938, Ann. Math. Stat., 9, 60

Woosley, S. E., \& Bloom, J. S. 2006, ARA\&A, 44, 507

Yassine, M., Piron, F., Mochkovitch, R., \& Daigne, F. 2017, A\&A, 606, A93

Yu, H.-F., van Eerten, H. J., Greiner, J., et al. 2015, A\&A, 583, A129

Zhang, B.-B., Uhm, Z. L., Connaughton, V., Briggs, M. S., \& Zhang, B. 2016, ApJ, 816, 72 
M. Yassine et al.: A new fitting function for GRB MeV spectra based on the internal shock synchrotron model

\section{Appendix A: Spectral analysis results}

Table A.1. Results of the Band and ISSM fits for GRB_B001 in the four time intervals.

\begin{tabular}{lcccccc}
\hline \hline Time interval & Model & $E_{\mathrm{p}}$ & $E_{\mathrm{p}} / E_{\mathrm{p}, \text { rue }}$ & $\alpha$ & $\begin{array}{c}\beta \\
\left(\times 10^{-3} \mathrm{~cm}^{-2} \mathrm{~s}^{-1} \mathrm{keV}^{-1}\right)\end{array}$ & Amplitude \\
\hline$[0 \mathrm{~s}, 1 \mathrm{~s}]$ & Band & $761 \pm 14$ & 0.66 & $-1.14 \pm 0.01$ & $-2.16 \pm 0.01$ & $1962 \pm 10$ \\
& ISSM & $1216 \pm 25$ & 1.06 & $-1.07 \pm 0.01$ & $-2.45 \pm 0.02$ & $36 \pm 1$ \\
\hline$[1 \mathrm{~s}, 3 \mathrm{~s}]$ & Band & $295 \pm 3$ & 0.62 & $-1.22 \pm 0.01$ & $-2.13 \pm 0.01$ & $3100 \pm 19$ \\
& ISSM & $459 \pm 6$ & 0.96 & $-1.09 \pm 0.01$ & $-2.35 \pm 0.01$ & $145 \pm 1$ \\
\hline$[3 \mathrm{~s}, 6 \mathrm{~s}]$ & Band & $99 \pm 3$ & 0.87 & $-1.37 \pm 0.02$ & $-2.16 \pm 0.01$ & $477 \pm 15$ \\
& ISSM & $119 \pm 3$ & 1.04 & $-0.95 \pm 0.08$ & $-2.29 \pm 0.02$ & $192 \pm 1$ \\
\hline$[0 \mathrm{~s}, 6 \mathrm{~s}]$ & Band & $378 \pm 4$ & 0.51 & $-1.27 \pm 0.01$ & $-2.09 \pm 0.01$ & $1465 \pm 6$ \\
& ISSM & $659 \pm 9$ & 0.88 & $-1.16 \pm 0.01$ & $-2.28 \pm 0.01$ & $34 \pm 1$ \\
\hline
\end{tabular}

Notes. The true peak energies are $E_{\mathrm{p}, \text { true }}=1150,478,114$ and $745 \mathrm{keV}$ for the time intervals $[0 \mathrm{~s}, 1 \mathrm{~s}],[1 \mathrm{~s}, 3 \mathrm{~s}],[3 \mathrm{~s}, 6 \mathrm{~s}]$ and $[0 \mathrm{~s}, 6 \mathrm{~s}]$ respectively.

Table A.2. Results of the Band and ISSM fits for GRB_B010 in the four time intervals.

\begin{tabular}{lcccccc}
\hline \hline Time interval & Model & $E_{\mathrm{p}}$ & $E_{\mathrm{p}} / E_{\mathrm{p}, \text { true }}$ & $\alpha$ & $\begin{array}{c}\beta \\
\left(\times 10^{-3} \mathrm{~cm}^{-2} \mathrm{~s}^{-1} \mathrm{keV}^{-1}\right)\end{array}$ & Amplitude \\
\hline$[0 \mathrm{~s}, 1 \mathrm{~s}]$ & Band & $677 \pm 40$ & 0.59 & $-1.13 \pm 0.01$ & $-2.11 \pm 0.04$ & $2008 \pm 37$ \\
& ISSM & $1178 \pm 82$ & 1.02 & $-1.06 \pm 0.02$ & $-2.41 \pm 0.07$ & $35 \pm 1$ \\
\hline$[1 \mathrm{~s}, 3 \mathrm{~s}]$ & Band & $298 \pm 11$ & 0.62 & $-1.23 \pm 0.01$ & $-2.13 \pm 0.02$ & $3077 \pm 58$ \\
& ISSM & $470 \pm 18$ & 0.98 & $-1.08 \pm 0.03$ & $-2.33 \pm 0.03$ & $145 \pm 2$ \\
\hline$[3 \mathrm{~s}, 6 \mathrm{~s}]$ & Band & $104 \pm 11$ & 0.91 & $-1.44 \pm 0.05$ & $-2.13 \pm 0.03$ & $424 \pm 41$ \\
& ISSM & $127 \pm 11$ & 1.11 & $-1.02 \pm 0.27$ & $-2.23 \pm 0.05$ & $187 \pm 3$ \\
\hline$[0 \mathrm{~s}, 6 \mathrm{~s}]$ & Band & $377 \pm 13$ & 0.51 & $-1.27 \pm 0.01$ & $-2.07 \pm 0.02$ & $1456 \pm 20$ \\
& ISSM & $685 \pm 32$ & 0.92 & $-1.16 \pm 0.02$ & $-2.26 \pm 0.03$ & $34 \pm 1$ \\
\hline
\end{tabular}

Notes. The true peak energies are $E_{\mathrm{p}, \text { true }}=1150,478,114$ and $745 \mathrm{keV}$ for the time intervals $[0 \mathrm{~s}, 1 \mathrm{~s}],[1 \mathrm{~s}, 3 \mathrm{~s}],[3 \mathrm{~s}, 6 \mathrm{~s}]$ and $[0 \mathrm{~s}, 6 \mathrm{~s}]$ respectively.

Table A.3. Results of the Band and ISSM fits for GRB_B100 in the four time intervals.

\begin{tabular}{lcccccc}
\hline \hline Time interval & Model & $E_{\mathrm{p}}$ & $E_{\mathrm{p}} / E_{\mathrm{p}, \text { true }}$ & $\alpha$ & $\begin{array}{c}\beta \\
\left(\times 10^{-3} \mathrm{~cm}^{-2} \mathrm{~s}^{-1} \mathrm{keV}^{-1}\right)\end{array}$ & Amplitude \\
\hline$[0 \mathrm{~s}, 1 \mathrm{~s}]$ & Band & $709 \pm 135$ & 0.62 & $-1.09 \pm 0.05$ & $-2.07 \pm 0.12$ & $193 \pm 11$ \\
& ISSM & $1333 \pm 324$ & 1.16 & $-1.03 \pm 0.08$ & $-2.40 \pm 0.24$ & $4 \pm 1$ \\
\hline$[1 \mathrm{~s}, 3 \mathrm{~s}]$ & Band & $297 \pm 37$ & 0.62 & $-1.25 \pm 0.04$ & $-2.13 \pm 0.07$ & $301 \pm 18$ \\
& ISSM & $457 \pm 50$ & 0.96 & $-1.18 \pm 0.07$ & $-2.45 \pm 0.16$ & $14 \pm 1$ \\
\hline$[3 \mathrm{~s}, 6 \mathrm{~s}]$ & Band & $55 \pm 17$ & 0.48 & $-0.90 \pm 0.38$ & $-2.00 \pm 0.06$ & $136 \pm 121$ \\
& ISSM & $130 \pm 34$ & 1.14 & Unconstrained & $-2.18 \pm 0.13$ & $19 \pm 1$ \\
\hline$[0 \mathrm{~s}, 6 \mathrm{~s}]$ & Band & $445 \pm 50$ & 0.60 & $-1.31 \pm 0.03$ & $-2.13 \pm 0.07$ & $137 \pm 5$ \\
& ISSM & $678 \pm 78$ & 0.91 & $-1.24 \pm 0.04$ & $-2.38 \pm 0.12$ & $3.5 \pm 0.1$ \\
\hline
\end{tabular}

Notes. The true peak energies are $E_{\mathrm{p}, \text { true }}=1150,478,114$ and $745 \mathrm{keV}$ for the time intervals $[0 \mathrm{~s}, 1 \mathrm{~s}],[1 \mathrm{~s}, 3 \mathrm{~s}],[3 \mathrm{~s}, 6 \mathrm{~s}]$ and $[0 \mathrm{~s}, 6 \mathrm{~s}]$ respectively. 
Table A.4. Results of the Band and ISSM spectral fits to GBM data for the prompt emission of 74 GRBs.

\begin{tabular}{|c|c|c|c|c|c|c|c|c|}
\hline GRB name & Models & $E_{\mathrm{p}}$ & $\alpha$ & $\alpha_{10}$ & $\beta$ & $\beta_{\mathrm{b}}$ & Amplitude & $\mathrm{PG}_{\text {stat }} /$ d.o.f. \\
\hline \multirow[t]{2}{*}{ GRB080817161 } & Band & $410 \pm 14$ & $-0.96 \pm 0.01$ & $-1.00 \pm 0.01$ & $-2.32 \pm 0.08$ & $-2.32 \pm 0.08$ & $145 \pm 2$ & $1031 / 469$ \\
\hline & ISSM & $509 \pm 11$ & $-0.88 \pm 0.02$ & $-0.93 \pm 0.01$ & $-3.13 \pm 0.25$ & $-2.03 \pm 0.02$ & $8.9 \pm 0.2$ & $1021 / 469$ \\
\hline \multirow[t]{2}{*}{ GRB080825593 } & Band & $187 \pm 7$ & $-0.64 \pm 0.03$ & $-0.75 \pm 0.02$ & $-2.35 \pm 0.10$ & $-2.35 \pm 0.10$ & $641 \pm 30$ & $1144 / 469$ \\
\hline & ISSM & $211 \pm 5$ & $-0.56 \pm 0.06$ & $-0.66 \pm 0.04$ & $-5.19 \pm 1.22$ & $-2.11 \pm 0.03$ & $10.6 \pm 0.2$ & $1149 / 469$ \\
\hline \multirow[t]{2}{*}{ GRB081125496 } & Band & $183 \pm 8$ & $-0.51 \pm 0.05$ & $-0.62 \pm 0.04$ & $-3.00 \pm 0.92$ & $-3.00 \pm 0.92$ & $913 \pm 72$ & $534 / 351$ \\
\hline & ISSM & $187 \pm 6$ & $-0.40 \pm 0.09$ & $-0.51 \pm 0.07$ & $-7.06 \pm 2.56$ & $-2.67 \pm 0.10$ & $10.2 \pm 0.7$ & $532 / 351$ \\
\hline \multirow[t]{2}{*}{ GRB081207680 } & Band & $705 \pm 40$ & $-0.77 \pm 0.02$ & $-0.80 \pm 0.02$ & $-2.62 \pm 0.28$ & $-2.62 \pm 0.28$ & $75 \pm 1$ & $1794 / 353$ \\
\hline & ISSM & $868 \pm 39$ & $-0.69 \pm 0.04$ & $-0.72 \pm 0.03$ & $-3.37 \pm 0.28$ & $-2.14 \pm 0.04$ & $8.6 \pm 0.2$ & $1777 / 353$ \\
\hline \multirow[t]{2}{*}{ GRB081224887 } & Band & $404 \pm 10$ & $-0.71 \pm 0.01$ & $-0.75 \pm 0.01$ & $-9.09 \pm 1.58$ & $-9.09 \pm 1.58$ & $372 \pm 6$ & $648 / 474$ \\
\hline & ISSM & $411 \pm 7$ & $-0.67 \pm 0.01$ & $-0.71 \pm 0.01$ & $-10.00 \pm 1.50$ & $-5.47 \pm 0.03$ & $23.8 \pm 0.3$ & $647 / 474$ \\
\hline \multirow[t]{2}{*}{ GRB090328401 } & Band & $754 \pm 51$ & $-1.05 \pm 0.02$ & $-1.07 \pm 0.01$ & $-2.44 \pm 0.19$ & $-2.44 \pm 0.19$ & $98 \pm 2$ & $1241 / 473$ \\
\hline & ISSM & $897 \pm 80$ & $-1.04 \pm 0.02$ & $-1.05 \pm 0.02$ & $-4.37 \pm 1.42$ & $-2.15 \pm 0.04$ & $9.6 \pm 0.2$ & $1243 / 473$ \\
\hline \multirow[t]{2}{*}{ GRB090528516 } & Band & $154 \pm 7$ & $-0.84 \pm 0.04$ & $-0.95 \pm 0.04$ & $-2.04 \pm 0.05$ & $-2.04 \pm 0.05$ & $197 \pm 14$ & $2652 / 472$ \\
\hline & ISSM & $241 \pm 24$ & $-0.57 \pm 0.14$ & $-0.76 \pm 0.08$ & $-2.55 \pm 0.18$ & $-1.82 \pm 0.04$ & $3.9 \pm 0.2$ & $2650 / 472$ \\
\hline \multirow[t]{2}{*}{ GRB090618353 } & Band & $164 \pm 3$ & $-1.10 \pm 0.01$ & $-1.18 \pm 0.01$ & $-2.46 \pm 0.04$ & $-2.46 \pm 0.04$ & $720 \pm 15$ & $1229 / 238$ \\
\hline & ISSM & $171 \pm 2$ & $-0.93 \pm 0.03$ & $-1.04 \pm 0.02$ & $-3.15 \pm 0.11$ & $-2.21 \pm 0.01$ & $13.0 \pm 0.2$ & $1173 / 238$ \\
\hline \multirow[t]{2}{*}{ GRB090718762 } & Band & $170 \pm 5$ & $-1.11 \pm 0.01$ & $-1.19 \pm 0.01$ & $-2.69 \pm 0.18$ & $-2.69 \pm 0.18$ & $312 \pm 8$ & $666 / 469$ \\
\hline & ISSM & $173 \pm 2$ & $-1.02 \pm 0.02$ & $-1.10 \pm 0.01$ & $-4.22 \pm 0.57$ & $-2.41 \pm 0.03$ & $5.3 \pm 0.3$ & $662 / 469$ \\
\hline \multirow[t]{2}{*}{ GRB090719063 } & Band & $240 \pm 2$ & $-0.54 \pm 0.02$ & $-0.62 \pm 0.02$ & $-2.95 \pm 0.12$ & $-2.95 \pm 0.12$ & $1281 \pm 30$ & $460 / 354$ \\
\hline & ISSM & $250 \pm 4$ & $-0.45 \pm 0.03$ & $-0.53 \pm 0.03$ & $-6.62 \pm 0.94$ & $-2.59 \pm 0.03$ & $30.9 \pm 0.6$ & $455 / 354$ \\
\hline \multirow[t]{2}{*}{ GRB090809978 } & Band & $175 \pm 10$ & $-0.74 \pm 0.03$ & $-0.84 \pm 0.02$ & $-1.98 \pm 0.04$ & $-1.98 \pm 0.04$ & $677 \pm 35$ & $815 / 471$ \\
\hline & ISSM & $344 \pm 46$ & $-0.40 \pm 0.10$ & $-0.62 \pm 0.06$ & $-2.37 \pm 0.09$ & $-1.75 \pm 0.02$ & $16.7 \pm 0.5$ & $810 / 471$ \\
\hline \multirow[t]{2}{*}{ GRB090829672 } & Band & $196 \pm 9$ & $-1.42 \pm 0.01$ & $-1.46 \pm 0.01$ & $-2.36 \pm 0.10$ & $-2.36 \pm 0.10$ & $280 \pm 8$ & $510 / 237$ \\
\hline & ISSM & $208 \pm 10$ & $-1.33 \pm 0.04$ & $-1.39 \pm 0.03$ & $-2.64 \pm 0.17$ & $-2.14 \pm 0.02$ & $8.0 \pm 0.2$ & $498 / 237$ \\
\hline \multirow[t]{2}{*}{ GRB091003191 } & Band & $397 \pm 16$ & $-0.94 \pm 0.02$ & $-0.98 \pm 0.02$ & $-2.59 \pm 0.19$ & $-2.59 \pm 0.19$ & $272 \pm 7$ & $551 / 355$ \\
\hline & ISSM & $429 \pm 19$ & $-0.92 \pm 0.03$ & $-0.95 \pm 0.03$ & $-5.95 \pm 2.07$ & $-2.34 \pm 0.06$ & $16.1 \pm 0.5$ & $552 / 355$ \\
\hline GRB091120191 & Band & $136 \pm 5$ & $-1.16 \pm 0.03$ & $-1.25 \pm 0.02$ & $-2.92 \pm 0.28$ & $-2.92 \pm 0.28$ & $193 \pm 9$ & $965 / 470$ \\
\hline & ISSM & $134 \pm 4$ & $-1.08 \pm 0.02$ & $-1.17 \pm 0.01$ & $-4.83 \pm 1.89$ & $-2.61 \pm 0.04$ & $2.1 \pm 0.3$ & $964 / 470$ \\
\hline GRB091128285 & Band & $192 \pm 1$ & $-0.95 \pm 0.01$ & $-1.03 \pm 0.01$ & $-2.58 \pm 0.16$ & $-2.58 \pm 0.16$ & $160 \pm 1$ & $1037 / 353$ \\
\hline & ISSM & $199 \pm 2$ & $-0.92 \pm 0.02$ & $-0.98 \pm 0.01$ & $-6.62 \pm 1.66$ & $-2.40 \pm 0.03$ & $2.8 \pm 0.0$ & $1041 / 353$ \\
\hline GRB100322045 & Band & $333 \pm 10$ & $-0.88 \pm 0.01$ & $-0.93 \pm 0.01$ & $-2.20 \pm 0.04$ & $-2.20 \pm 0.04$ & $307 \pm 6$ & $779 / 469$ \\
\hline & ISSM & $487 \pm 23$ & $-0.69 \pm 0.03$ & $-0.78 \pm 0.02$ & $-2.60 \pm 0.07$ & $-1.91 \pm 0.02$ & $15.6 \pm 0.2$ & $726 / 469$ \\
\hline GRB100324172 & Band & $461 \pm 12$ & $-0.58 \pm 0.02$ & $-0.62 \pm 0.02$ & $-5.60 \pm 1.46$ & $-5.60 \pm 1.46$ & $369 \pm 6$ & $627 / 469$ \\
\hline & ISSM & $468 \pm 9$ & $-0.54 \pm 0.02$ & $-0.58 \pm 0.02$ & $-10.00 \pm 1.50$ & $-4.21 \pm 0.04$ & $30.5 \pm 0.5$ & $631 / 469$ \\
\hline GRB100414097 & Band & $637 \pm 12$ & $-0.53 \pm 0.01$ & $-0.56 \pm 0.01$ & $-4.95 \pm 1.86$ & $-4.95 \pm 1.86$ & $349 \pm 4$ & $1070 / 471$ \\
\hline & ISSM & $651 \pm 12$ & $-0.49 \pm 0.01$ & $-0.52 \pm 0.01$ & $-10.00 \pm 5.00$ & $-3.88 \pm 0.03$ & $46.1 \pm 0.3$ & $1090 / 471$ \\
\hline GRB100511035 & Band & $625 \pm 38$ & $-1.28 \pm 0.01$ & $-1.29 \pm 0.01$ & $-9.37 \pm 1.37$ & $-9.37 \pm 1.37$ & $94 \pm 2$ & $798 / 473$ \\
\hline & ISSM & $656 \pm 51$ & $-1.28 \pm 0.01$ & $-1.29 \pm 0.01$ & $-10.00 \pm 1.50$ & $-5.56 \pm 0.02$ & $6.8 \pm 0.1$ & $798 / 473$ \\
\hline GRB100612726 & Band & $113 \pm 2$ & $-0.57 \pm 0.04$ & $-0.75 \pm 0.03$ & $-2.55 \pm 0.07$ & $-2.55 \pm 0.07$ & $1290 \pm 79$ & $524 / 472$ \\
\hline & ISSM & $121 \pm 2$ & $-0.25 \pm 0.04$ & $-0.51 \pm 0.02$ & $-3.80 \pm 0.32$ & $-2.23 \pm 0.02$ & $6.3 \pm 0.6$ & $528 / 472$ \\
\hline GRB100707032 & Band & $266 \pm 14$ & $-0.69 \pm 0.03$ & $-0.76 \pm 0.02$ & $-2.08 \pm 0.05$ & $-2.08 \pm 0.05$ & $236 \pm 10$ & $450 / 236$ \\
\hline & ISSM & $504 \pm 61$ & $-0.36 \pm 0.08$ & $-0.52 \pm 0.06$ & $-2.39 \pm 0.10$ & $-1.79 \pm 0.02$ & $9.7 \pm 0.2$ & $440 / 236$ \\
\hline GRB100719989 & Band & $321 \pm 12$ & $-0.69 \pm 0.03$ & $-0.74 \pm 0.02$ & $-2.41 \pm 0.08$ & $-2.41 \pm 0.08$ & $462 \pm 15$ & $733 / 354$ \\
\hline & ISSM & $384 \pm 13$ & $-0.56 \pm 0.04$ & $-0.63 \pm 0.03$ & $-3.55 \pm 0.34$ & $-2.07 \pm 0.03$ & $22.4 \pm 0.4$ & $726 / 354$ \\
\hline GRB100826957 & Band & $461 \pm 25$ & $-1.05 \pm 0.01$ & $-1.08 \pm 0.01$ & $-2.05 \pm 0.02$ & $-2.05 \pm 0.02$ & $310 \pm 5$ & $717 / 237$ \\
\hline & ISSM & $1005 \pm 77$ & $-0.95 \pm 0.02$ & $-1.00 \pm 0.02$ & $-2.30 \pm 0.04$ & $-1.80 \pm 0.02$ & $21.1 \pm 0.2$ & $696 / 237$ \\
\hline GRB100829876 & Band & $136 \pm 5$ & $-0.60 \pm 0.08$ & $-0.75 \pm 0.06$ & $-2.04 \pm 0.04$ & $-2.04 \pm 0.04$ & $946 \pm 104$ & $276 / 237$ \\
\hline & ISSM & $232 \pm 29$ & $-0.15 \pm 0.09$ & $-0.48 \pm 0.03$ & $-2.49 \pm 0.16$ & $-1.77 \pm 0.06$ & $13.8 \pm 0.8$ & $275 / 237$ \\
\hline GRB100910818 & Band & $159 \pm 10$ & $-0.94 \pm 0.01$ & $-1.03 \pm 0.00$ & $-2.46 \pm 0.11$ & $-2.46 \pm 0.11$ & $376 \pm 8$ & $587 / 469$ \\
\hline & ISSM & $168 \pm 2$ & $-0.84 \pm 0.03$ & $-0.94 \pm 0.02$ & $-4.42 \pm 0.78$ & $-2.25 \pm 0.03$ & $5.1 \pm 0.4$ & $586 / 469$ \\
\hline GRB100918863 & Band & $562 \pm 3$ & $-0.80 \pm 0.01$ & $-0.84 \pm 0.00$ & $-2.74 \pm 0.12$ & $-2.74 \pm 0.12$ & $205 \pm 1$ & $709 / 352$ \\
\hline & ISSM & $612 \pm 10$ & $-0.76 \pm 0.01$ & $-0.79 \pm 0.01$ & $-5.05 \pm 0.14$ & $-2.37 \pm 0.02$ & $18.9 \pm 0.2$ & $714 / 352$ \\
\hline GRB101014175 & Band & $210 \pm 4$ & $-1.17 \pm 0.01$ & $-1.22 \pm 0.01$ & $-2.79 \pm 0.11$ & $-2.79 \pm 0.11$ & $625 \pm 12$ & $356 / 237$ \\
\hline & ISSM & $218 \pm 5$ & $-1.16 \pm 0.01$ & $-1.20 \pm 0.01$ & $-9.04 \pm 3.19$ & $-2.61 \pm 0.02$ & $14.6 \pm 0.4$ & $365 / 237$ \\
\hline GRB101023951 & Band & $185 \pm 7$ & $-1.22 \pm 0.03$ & $-1.28 \pm 0.02$ & $-2.58 \pm 0.14$ & $-2.58 \pm 0.14$ & $220 \pm 10$ & $1653 / 353$ \\
\hline & ISSM & $187 \pm 6$ & $-1.12 \pm 0.03$ & $-1.19 \pm 0.02$ & $-3.61 \pm 0.48$ & $-2.33 \pm 0.04$ & $4.7 \pm 0.2$ & $1649 / 353$ \\
\hline GRB101126198 & Band & $135 \pm 1$ & $-1.29 \pm 0.01$ & $-1.37 \pm 0.00$ & $-2.65 \pm 0.17$ & $-2.65 \pm 0.17$ & $211 \pm 1$ & $890 / 470$ \\
\hline & ISSM & $140 \pm 1$ & $-1.28 \pm 0.01$ & $-1.34 \pm 0.01$ & $-8.81 \pm 0.29$ & $-2.51 \pm 0.01$ & $2.5 \pm 0.1$ & $893 / 470$ \\
\hline GRB101231067 & Band & $214 \pm 2$ & $-0.75 \pm 0.02$ & $-0.83 \pm 0.01$ & $-9.99 \pm 4.99$ & $-9.99 \pm 4.99$ & $251 \pm 3$ & $531 / 353$ \\
\hline & ISSM & $216 \pm 4$ & $-0.70 \pm 0.04$ & $-0.78 \pm 0.03$ & $-8.07 \pm 2.97$ & $-5.19 \pm 0.07$ & $4.6 \pm 0.2$ & $531 / 353$ \\
\hline GRB110301214 & Band & $110 \pm 1$ & $-0.83 \pm 0.02$ & $-0.99 \pm 0.02$ & $-2.73 \pm 0.05$ & $-2.73 \pm 0.05$ & $4242 \pm 124$ & $713 / 470$ \\
\hline & ISSM & $110 \pm 2$ & $-0.59 \pm 0.04$ & $-0.80 \pm 0.03$ & $-4.03 \pm 0.18$ & $-2.42 \pm 0.01$ & $22.0 \pm 0.9$ & $690 / 470$ \\
\hline
\end{tabular}

Notes. The amplitudes are given in units of $10^{-4} \mathrm{~cm}^{-2} \mathrm{~s}^{-1} \mathrm{keV}^{-1}$. 
Table A.4. continued.

\begin{tabular}{|c|c|c|c|c|c|c|c|c|}
\hline GRB name & Models & $E_{\mathrm{p}}$ & $\alpha$ & $\alpha_{10}$ & $\beta$ & $\beta_{\mathrm{b}}$ & Amplitude & $\mathrm{PG}_{\text {stat }} /$ d.o.f. \\
\hline \multirow{2}{*}{ GRB110622158 } & Band & $105 \pm 1$ & $-0.64 \pm 0.03$ & $-0.83 \pm 0.02$ & $-2.44 \pm 0.04$ & $-2.44 \pm 0.04$ & $541 \pm 25$ & $1973 / 471$ \\
\hline & ISSM & $114 \pm 2$ & $-0.17 \pm 0.06$ & $-0.52 \pm 0.03$ & $-3.28 \pm 0.14$ & $-2.15 \pm 0.01$ & $2.9 \pm 0.1$ & $1997 / 471$ \\
\hline \multirow{2}{*}{ GRB110625881 } & Band & $179 \pm 4$ & $-0.77 \pm 0.02$ & $-0.87 \pm 0.01$ & $-2.33 \pm 0.04$ & $-2.33 \pm 0.04$ & $929 \pm 26$ & $1285 / 470$ \\
\hline & ISSM & $210 \pm 3$ & $-0.53 \pm 0.05$ & $-0.68 \pm 0.03$ & $-3.16 \pm 0.14$ & $-2.05 \pm 0.01$ & $17.4 \pm 0.3$ & $1250 / 470$ \\
\hline \multirow[t]{2}{*}{ GRB110717319 } & Band & $376 \pm 5$ & $-1.01 \pm 0.01$ & $-1.05 \pm 0.01$ & $-9.37 \pm 1.57$ & $-9.37 \pm 1.57$ & $98 \pm 1$ & $813 / 470$ \\
\hline & ISSM & $370 \pm 7$ & $-0.98 \pm 0.01$ & $-1.01 \pm 0.01$ & $-10.00 \pm 1.50$ & $-5.69 \pm 0.02$ & $5.1 \pm 0.1$ & $813 / 470$ \\
\hline \multirow[t]{2}{*}{ GRB110729142 } & Band & $307 \pm 11$ & $-1.02 \pm 0.02$ & $-1.07 \pm 0.01$ & $-2.21 \pm 0.15$ & $-2.21 \pm 0.15$ & $35 \pm 1$ & $838 / 473$ \\
\hline & ISSM & $390 \pm 26$ & $-0.91 \pm 0.07$ & $-0.97 \pm 0.06$ & $-2.89 \pm 0.31$ & $-1.98 \pm 0.03$ & $1.6 \pm 0.0$ & $835 / 473$ \\
\hline \multirow[t]{2}{*}{ GRB110731465 } & Band & $307 \pm 15$ & $-0.87 \pm 0.02$ & $-0.92 \pm 0.02$ & $-2.88 \pm 0.65$ & $-2.88 \pm 0.65$ & $565 \pm 14$ & $423 / 354$ \\
\hline & ISSM & $322 \pm 9$ & $-0.86 \pm 0.02$ & $-0.90 \pm 0.02$ & $-10.00 \pm 1.50$ & $-2.64 \pm 0.04$ & $23.3 \pm 0.6$ & $427 / 354$ \\
\hline \multirow[t]{2}{*}{ GRB110825102 } & Band & 26 & $-1.07 \pm 0.01$ & $-1.12 \pm 0.01$ & $-2.72 \pm 0.31$ & $-2.72 \pm 0.31$ & $177 \pm 1$ & $697 / 473$ \\
\hline & ISSM & $267 \pm 1$ & $-1.05 \pm 0.01$ & $-1.09 \pm 0.01$ & $-10.00 \pm 1.50$ & $-2.58 \pm 0.02$ & $5.6 \pm 0.0$ & $698 / 473$ \\
\hline \multirow[t]{2}{*}{ GRB110921912 } & Band & $513 \pm 20$ & $-0.88 \pm 0.01$ & $-0.91 \pm 0.01$ & $-2.36 \pm 0.09$ & $-2.36 \pm 0.09$ & $283 \pm 5$ & $506 / 356$ \\
\hline & ISSM & $678 \pm 43$ & $-0.78 \pm 0.04$ & $-0.82 \pm 0.03$ & $-2.89 \pm 0.19$ & $-2.00 \pm 0.03$ & $22.4 \pm 0.4$ & $489 / 356$ \\
\hline \multirow{2}{*}{ GRB111003465 } & Band & $205 \pm 7$ & $-0.95 \pm 0.02$ & $-1.02 \pm 0.02$ & $-2.43 \pm 0.10$ & $-2.43 \pm 0.10$ & $394 \pm 16$ & $627 / 473$ \\
\hline & ISSM & 228 & $-0.86 \pm 0.06$ & $-0.94 \pm 0.04$ & $-3.76 \pm 0.57$ & $-2.16 \pm 0.04$ & $9.3 \pm 0.4$ & $625 / 473$ \\
\hline \multirow[t]{2}{*}{ GRB111216389 } & Band & 165 & $-0.91 \pm 0.03$ & $-1.00 \pm 0.03$ & $-2.30 \pm 0.06$ & $-2.30 \pm 0.06$ & $199 \pm 9$ & $734 / 352$ \\
\hline & ISSM & 197 & $-0.79 \pm 0.07$ & $-0.90 \pm 0.05$ & $-3.30 \pm 0.30$ & $-2.04 \pm 0.02$ & $3.5 \pm 0.2$ & $730 / 352$ \\
\hline \multirow[t]{2}{*}{ GRB111220486 } & Band & $300 \pm 10$ & $-1.05 \pm 0.01$ & $-1.09 \pm 0.01$ & $-2.30 \pm 0.07$ & $-2.30 \pm 0.07$ & $308 \pm 6$ & $474 / 353$ \\
\hline & ISSM & $371 \pm 15$ & $-0.96 \pm 0.01$ & $-1.02 \pm 0.00$ & $-3.01 \pm 0.15$ & $-2.03 \pm 0.02$ & $13.2 \pm 0.2$ & $467 / 353$ \\
\hline \multirow[t]{2}{*}{ GRB120119170 } & Band & $208 \pm 1$ & $-1.03 \pm 0.01$ & $-1.09 \pm 0.01$ & $-2.54 \pm 0.10$ & $-2.54 \pm 0.10$ & $207 \pm 1$ & $774 / 469$ \\
\hline & ISSM & $226 \pm 3$ & $-0.97 \pm 0.01$ & $-1.04 \pm 0.01$ & $-4.37 \pm 0.30$ & $-2.27 \pm 0.02$ & $5.0 \pm 0.0$ & $773 / 469$ \\
\hline \multirow[t]{2}{*}{ GRB120129580 } & Band & & $-0.68 \pm 0.02$ & $-0.74 \pm 0.02$ & $-2.56 \pm 0.07$ & $-2.56 \pm 0.07$ & $3845 \pm 100$ & $392 / 236$ \\
\hline & ISSM & $337 \pm 8$ & $-0.47 \pm 0.03$ & $-0.56 \pm 0.03$ & $-3.28 \pm 0.18$ & $-2.16 \pm 0.02$ & $157.3 \pm 2.4$ & $346 / 236$ \\
\hline GRB120204054 & Band & & $-1.08 \pm$ & $-1.16 \pm$ & $-2.58 \pm 0.05$ & $-2.58 \pm 0.05$ & & $1763 / 470$ \\
\hline & ISSM & 171 & -1. & $-1.09 \pm 0$ & $-4.31 \pm$ & 0.01 & & $1760 / 470$ \\
\hline GRB120226871 & Band & $301 \pm 11$ & $-0.89 \pm 0.02$ & $-0.94 \pm 0.02$ & $-2.26 \pm 0.08$ & $-2.26 \pm 0.08$ & 231 & $1338 / 470$ \\
\hline & & & & & $-2.89 \pm 0.22$ & $-1.96 \pm 0.02$ & 10.3 & 470 \\
\hline G & $\mathrm{Ba}$ & 19 & -0 . & -0 . & $-2.00 \pm$ & -2 & & 471 \\
\hline & IS & $385 \pm$ & $-0.47 \pm 0.05$ & $-0.66 \pm 0.02$ & $-2.35 \pm 0.05$ & $-1.76 \pm 0.03$ & $22.7 \pm$ & $1357 / 471$ \\
\hline 090 & Band & $135 \pm 3$ & $-0.59 \pm 0.03$ & $-0.74 \pm 0.02$ & $-2.94 \pm 0.12$ & $-2.94 \pm 0.12$ & $4721 \pm 208$ & $524 / 352$ \\
\hline & ISSM & $132 \pm 3$ & $-0.28 \pm 0.07$ & $-0.49 \pm 0.05$ & $-4.49 \pm 0.42$ & $-2.55 \pm 0.03$ & $27.1 \pm 1.6$ & $501 / 352$ \\
\hline GRB120624933 & Band & $583 \pm 83$ & $-0.97 \pm 0.05$ & $-0.99 \pm 0.05$ & $-2.05 \pm 0.16$ & $-2.05 \pm 0.16$ & $18 \pm 1$ & $2055 / 469$ \\
\hline & ISSM & $1107 \pm 457$ & $-0.96 \pm 0.07$ & $-0.98 \pm 0.07$ & $-2.62 \pm 0.51$ & $-1.76 \pm 0.10$ & $1.6 \pm 0.1$ & $2058 / 469$ \\
\hline GRB120707800 & Band & $181 \pm 13$ & $-1.08 \pm 0.03$ & $-1.15 \pm 0.03$ & $-2.37 \pm 0.05$ & $-2.37 \pm 0.05$ & $708 \pm 29$ & $1173 / 352$ \\
\hline & ISSM & $189 \pm 6$ & $-0.76 \pm 0.10$ & $-0.91 \pm 0.19$ & $-2.83 \pm 0.13$ & $-2.14 \pm 0.06$ & $15.2 \pm 0.3$ & $1167 / 352$ \\
\hline GRB120711115 & Band & $1277 \pm 31$ & $-0.95 \pm 0.01$ & $-0.96 \pm 0.01$ & $-3.11 \pm 0.13$ & $-3.11 \pm 0.13$ & $385 \pm 2$ & $577 / 353$ \\
\hline & ISSM & $1360 \pm 27$ & $-0.95 \pm 0.01$ & $-0.96 \pm 0.01$ & $-8.68 \pm 1.64$ & $-2.75 \pm 0.02$ & $54.7 \pm 0.2$ & $594 / 353$ \\
\hline GRB130306991 & Band & $307 \pm 15$ & $-0.75 \pm 0.03$ & $-0.81 \pm 0.03$ & $-2.62 \pm 0.11$ & $-2.62 \pm 0.11$ & $301 \pm 6$ & $2204 / 470$ \\
\hline & & & $0 \pm 0.11$ & $-0.59 \pm 0.11$ & $-3.70 \pm 0.24$ & $-2.28 \pm 0.11$ & 12.6 & $2200 / 470$ \\
\hline GRB130327350 & Band & 375 & $-0.61 \pm 0.02$ & $-0.66 \pm 0.01$ & $-9.37 \pm 2.22$ & $-9.37 \pm 2.22$ & 287 & $1057 / 470$ \\
\hline & ISSM & 379 & $-0.57 \pm$ & $-0.61 \pm$ & $-10.00 \pm 1.50$ & $-5.54 \pm 0.03$ & 16.7 & $1063 / 470$ \\
\hline GRB130502327 & Band & $293 \pm 5$ & $-0.50 \pm 0.01$ & $-0.57 \pm 0.01$ & $-2.36 \pm 0.04$ & $-2.36 \pm 0.04$ & $972 \pm 16$ & $1361 / 473$ \\
\hline & ISSM & $354 \pm 5$ & $-0.35 \pm 0.02$ & $-0.44 \pm 0.02$ & $-3.72 \pm 0.16$ & $-2.02 \pm 0.01$ & $41.6 \pm 0.4$ & $1338 / 473$ \\
\hline GRB130504978 & Band & $654 \pm 29$ & $-1.20 \pm 0.01$ & $-1.21 \pm 0.01$ & $-2.27 \pm 0.07$ & $-2.27 \pm 0.07$ & $232 \pm 2$ & $2120 / 470$ \\
\hline & ISSM & $867 \pm 41$ & $-1.18 \pm 0.01$ & $-1.19 \pm 0.01$ & $-3.05 \pm 0.17$ & $-2.00 \pm 0.01$ & $18.1 \pm 0.2$ & $2125 / 470$ \\
\hline GRB130518580 & Band & $387 \pm 10$ & $-0.87 \pm 0.01$ & $-0.91 \pm 0.01$ & $-2.22 \pm 0.05$ & $-2.22 \pm 0.05$ & $330 \pm 6$ & $768 / 354$ \\
\hline & ISSM & $539 \pm 20$ & $-0.78 \pm 0.02$ & $-0.83 \pm 0.02$ & $-2.93 \pm 0.14$ & $-1.92 \pm 0.02$ & $20.3 \pm 0.2$ & $769 / 354$ \\
\hline GRB130606497 & Band & $515 \pm 21$ & $-1.13 \pm 0.01$ & $-1.15 \pm 0.01$ & $-2.10 \pm 0.02$ & $-2.10 \pm 0.02$ & $544 \pm 6$ & $892 / 236$ \\
\hline & ISSM & $926 \pm 43$ & $-1.03 \pm 0.01$ & $-1.07 \pm 0.01$ & $-2.35 \pm 0.04$ & $-1.86 \pm 0.01$ & $37.5 \pm 0.3$ & $919 / 236$ \\
\hline GRB130609902 & Band & $531 \pm 13$ & $-0.98 \pm 0.02$ & $-1.01 \pm 0.02$ & $-9.37 \pm 1.77$ & $-9.37 \pm 1.77$ & $47 \pm 1$ & $822 / 354$ \\
\hline & ISSM & $539 \pm 31$ & $-0.96 \pm 0.02$ & $-0.98 \pm 0.01$ & $-9.37 \pm 3.80$ & $-5.45 \pm 0.03$ & $3.7 \pm 0.1$ & $822 / 354$ \\
\hline GRB130720582 & Band & $65 \pm 3$ & $-0.95 \pm 0.03$ & $-1.18 \pm 0.03$ & $-2.39 \pm 0.02$ & $-2.39 \pm 0.02$ & $451 \pm 24$ & $2608 / 469$ \\
\hline & ISSM & $66 \pm 1$ & $-0.19 \pm 0.05$ & $-0.83 \pm 0.06$ & $-2.86 \pm 0.03$ & $-2.16 \pm 0.01$ & $1.7 \pm 0.0$ & $2570 / 469$ \\
\hline GRB131028076 & Band & $848 \pm 15$ & $-0.64 \pm 0.01$ & $-0.66 \pm 0.01$ & $-2.55 \pm 0.03$ & $-2.55 \pm 0.03$ & $791 \pm 6$ & $1132 / 353$ \\
\hline & ISSM & $952 \pm 9$ & $-0.61 \pm 0.00$ & $-0.63 \pm 0.00$ & $-6.16 \pm 0.22$ & $-2.24 \pm 0.01$ & $125.0 \pm 0.5$ & $2119 / 353$ \\
\hline GRB131118958 & Band & $332 \pm 14$ & $-0.69 \pm 0.02$ & $-0.75 \pm 0.02$ & $-9.37 \pm 1.67$ & $-9.37 \pm 1.67$ & $195 \pm 4$ & $1105 / 237$ \\
\hline & ISSM & $313 \pm 9$ & $-0.39 \pm 0.13$ & $-0.47 \pm 0.26$ & $-4.43 \pm 0.87$ & $-3.72 \pm 0.26$ & $8.6 \pm 0.2$ & $1092 / 237$ \\
\hline GRB131231198 & Band & $218 \pm 6$ & $-1.20 \pm 0.01$ & $-1.25 \pm 0.01$ & $-2.41 \pm 0.04$ & $-2.41 \pm 0.04$ & $1119 \pm 18$ & $1350 / 355$ \\
\hline & ISSM & $232 \pm 4$ & $-1.08 \pm 0.02$ & $-1.15 \pm 0.05$ & $-3.10 \pm 0.13$ & $-2.17 \pm 0.05$ & $31.5 \pm 0.3$ & $1315 / 355$ \\
\hline GRB140306146 & Band & $1529 \pm 73$ & $-1.01 \pm 0.01$ & $-1.02 \pm 0.01$ & $-5.09 \pm 1.80$ & $-5.09 \pm 1.80$ & $126 \pm 1$ & $1492 / 355$ \\
\hline & ISSM & $1535 \pm 62$ & $-1.00 \pm 0.01$ & $-1.01 \pm 0.01$ & $-10.00 \pm 1.50$ & $-4.05 \pm 0.02$ & $17.7 \pm 0.2$ & $1495 / 355$ \\
\hline GRB140416060 & Band & $97 \pm 3$ & $-1.15 \pm 0.01$ & $-1.27 \pm 0.01$ & $-2.37 \pm 0.03$ & $-2.37 \pm 0.03$ & $1056 \pm 75$ & $2323 / 237$ \\
\hline & ISSM & $101 \pm 3$ & $-0.86 \pm 0.06$ & $-1.08 \pm 0.12$ & $-2.93 \pm 0.11$ & $-2.16 \pm 0.06$ & $9.8 \pm 0.2$ & $2315 / 237$ \\
\hline
\end{tabular}


Table A.4. continued.

\begin{tabular}{|c|c|c|c|c|c|c|c|c|}
\hline GRB name & Models & $E_{\mathrm{p}}$ & $\alpha$ & $\alpha_{10}$ & $\beta$ & $\beta_{\mathrm{b}}$ & Amplitude & $\mathrm{PG}_{\text {stat }} /$ d.o.f. \\
\hline \multirow[t]{2}{*}{ GRB140508128 } & Band & $264 \pm 13$ & $-1.01 \pm 0.02$ & $-1.07 \pm 0.02$ & $-2.11 \pm 0.04$ & $-2.11 \pm 0.04$ & $312 \pm 11$ & $1153 / 235$ \\
\hline & ISSM & $434 \pm 44$ & $-0.83 \pm 0.06$ & $-0.93 \pm 0.04$ & $-2.41 \pm 0.10$ & $-1.87 \pm 0.02$ & $12.4 \pm 0.2$ & $1164 / 235$ \\
\hline \multirow[t]{2}{*}{ GRB140523129 } & Band & $269 \pm 7$ & $-0.90 \pm 0.01$ & $-0.96 \pm 0.01$ & $-2.69 \pm 0.13$ & $-2.69 \pm 0.13$ & $632 \pm 9$ & $765 / 471$ \\
\hline & ISSM & $285 \pm 4$ & $-0.83 \pm 0.01$ & $-0.89 \pm 0.01$ & $-4.71 \pm 0.37$ & $-2.38 \pm 0.01$ & $21.4 \pm 0.3$ & $760 / 471$ \\
\hline \multirow[t]{2}{*}{ GRB140810782 } & Band & $309 \pm 6$ & $-0.88 \pm 0.01$ & $-0.93 \pm 0.01$ & $-2.41 \pm 0.06$ & $-2.41 \pm 0.06$ & $286 \pm 5$ & $896 / 353$ \\
\hline & ISSM & $368 \pm 14$ & $-0.75 \pm 0.03$ & $-0.81 \pm 0.03$ & $-3.17 \pm 0.20$ & $-2.08 \pm 0.03$ & $12.6 \pm 0.2$ & $871 / 353$ \\
\hline \multirow[t]{2}{*}{ GRB150118409 } & Band & $763 \pm 17$ & $-0.84 \pm 0.01$ & $-0.86 \pm 0.01$ & $-3.51 \pm 0.25$ & $-3.51 \pm 0.25$ & $332 \pm 3$ & $2545 / 469$ \\
\hline & ISSM & $795 \pm 18$ & $-0.83 \pm 0.01$ & $-0.84 \pm 0.01$ & $-10.00 \pm 1.50$ & $-3.07 \pm 0.02$ & $39.9 \pm 0.3$ & $2558 / 469$ \\
\hline \multirow[t]{2}{*}{ GRB150330828 } & Band & $265 \pm 5$ & $-1.01 \pm 0.01$ & $-1.06 \pm 0.01$ & $-2.25 \pm 0.04$ & $-2.25 \pm 0.04$ & $202 \pm 3$ & $1708 / 469$ \\
\hline & ISSM & $346 \pm$ & $-0.90 \pm 0.02$ & $-0.97 \pm 0.02$ & $-2.86 \pm 0.13$ & $-1.98 \pm 0.01$ & $7.7 \pm 0.1$ & $1683 / 469$ \\
\hline \multirow[t]{2}{*}{ GRB150403913 } & Band & $402 \pm 16$ & $-0.82 \pm 0.02$ & $-0.86 \pm 0.02$ & $-2.09 \pm 0.04$ & $-2.09 \pm 0.04$ & $437 \pm 10$ & $624 / 355$ \\
\hline & ISSM & $721 \pm 45$ & $-0.67 \pm 0.03$ & $-0.74 \pm 0.02$ & $-2.49 \pm 0.07$ & $-1.80 \pm 0.02$ & $29.0 \pm 0.4$ & $578 / 355$ \\
\hline \multirow[t]{2}{*}{ GRB150627183 } & Band & $243 \pm 5$ & $-0.92 \pm 0.01$ & $-0.98 \pm 0.01$ & $-2.19 \pm 0.02$ & $-2.19 \pm 0.02$ & $664 \pm 11$ & $1109 / 355$ \\
\hline & ISSM & & & & $-2.73 \pm 0.07$ & $-1.93 \pm 0.01$ & $23.0 \pm 0.2$ & $1057 / 355$ \\
\hline \multirow[t]{2}{*}{ GRB150902733 } & Band & $368 \pm 7$ & $-0.49 \pm 0.01$ & $-0.55 \pm 0.01$ & $-2.35 \pm 0.04$ & $-2.35 \pm 0.04$ & $1085 \pm 17$ & $761 / 470$ \\
\hline & ISSM & $472 \pm 7$ & $-0.30 \pm 0.03$ & $-0.38 \pm 0.02$ & $-3.24 \pm 0.10$ & $-1.97 \pm 0.01$ & $68.3 \pm 0.6$ & $656 / 470$ \\
\hline \multirow[t]{2}{*}{ GRB160802259 } & Band & $295 \pm 5$ & $-0.54 \pm 0.02$ & $-0.62 \pm 0.02$ & $-2.47 \pm 0.07$ & $-2.47 \pm 0.07$ & $863 \pm 20$ & $314 / 237$ \\
\hline & ISSM & $346 \pm 9$ & $-0.40 \pm 0.01$ & $-0.49 \pm 0.01$ & $-3.73 \pm 0.13$ & $-2.10 \pm 0.02$ & $36.2 \pm 0.7$ & $298 / 237$ \\
\hline \multirow[t]{2}{*}{ GRB160905471 } & Band & $1063 \pm 52$ & $-0.89 \pm 0.01$ & $-0.90 \pm 0.01$ & $-3.01 \pm 0.27$ & $-3.01 \pm 0.27$ & $237 \pm 2$ & $730 / 356$ \\
\hline & ISSM & $1161 \pm 20$ & $-0.89 \pm 0.01$ & $-0.90 \pm 0.01$ & $-10.00 \pm 0.00$ & $-2.66 \pm 0.02$ & $33.5 \pm 0.2$ & $736 / 356$ \\
\hline \multirow[t]{2}{*}{ GRB160910722 } & Band & $335 \pm 7$ & $-0.76 \pm 0.01$ & $-0.82 \pm 0.01$ & $-2.23 \pm 0.03$ & $-2.23 \pm 0.03$ & $632 \pm 11$ & $786 / 469$ \\
\hline & ISSM & $460 \pm 11$ & $-0.60 \pm 0.02$ & $-0.67 \pm 0.04$ & $-2.85 \pm 0.08$ & $-1.92 \pm 0.02$ & $33.1 \pm 0.3$ & $746 / 469$ \\
\hline
\end{tabular}

